J

\title{
DIFFUSION CLIMATOLOGY FOR HYPOTHETICAL ACCIDENTS IN AREA 410 OF THE NEVADA TEST SITE
}

Reniall R. Petergon

Ifay 20, 1976

Prepared for U.S. Enorgy Researsh \& Development

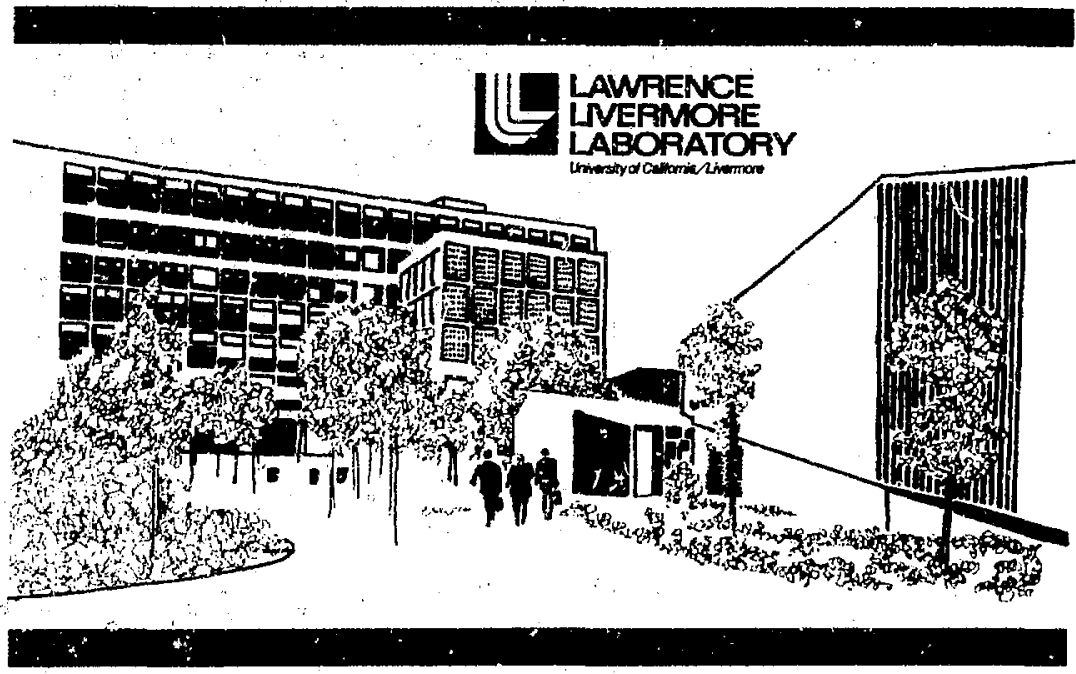


Nórex

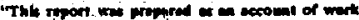

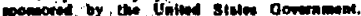

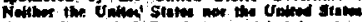

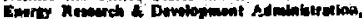

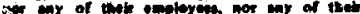

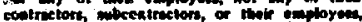

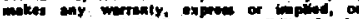

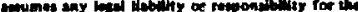

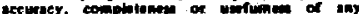

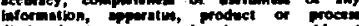

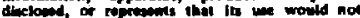

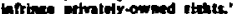

Printed in the United States of America Aralable :rom.

National Techivical Infermation Service U.S. Depurtment of Commerce 5285 Port Royal Row

Springfield, VA 2216!

Price: Ptinted Copy 5 ; Microflche \$2.25

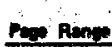

\section{Dowintie}

Prito

53.50

4.00

4.50

5.00

5,25

5.50

6.00

7.50

7.75

8.00

9.00

9.25

9.75

\footnotetext{
i Mlcrollche $\$ 2.25$
}

\section{Pow}

Pas Rans

Domint:

부술

$326-350$

10.00

$351-375$

10.50

10.75

11.00

$401-425$

11.75

12.00

12,50

12.75

13.00

13.50

13.75

251-275

276-300

$301-325$

.
451-475

476-500

501-\$25

$526-550$

$551-575$

$576-600$

601-up $\bullet$

Add 52.50. for wach additoral 100 pere inctitimat from 691 to I,000 phes:

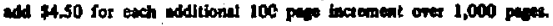




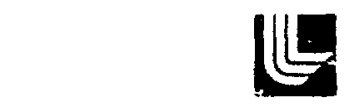

\section{LAWRENCE LVEAMOAE LABORATORY}

Univarsily of Ciatomi Linamere Cuttoma 94550

\section{ICK!-5207: \\ DIFFUSION CLIMATOLOGY FOR HYPOTHETICAL ACCIDENTS IN AREA 410 OF THE NEVADA TEST SITE}

Kondall H. Heterson

4ar 20, 1976

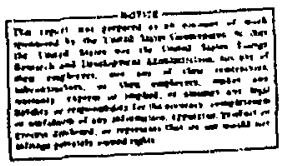




\section{Contents}

Abntract . . . . . . . . . . . . . . . . . . . . . . 1

Introduct tón . . . . . . . . . . . . . . . . . . . . . . . . 1

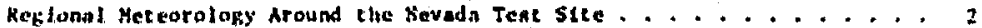

fieneral climule . . . . . . . . . . . . . . . . . . 2

Sevore heather . , . . . . . . . . . . . . . . . . . . . 2

Atr-Pollution Potental at ats . . . . . . . . . . . . . . . . 4

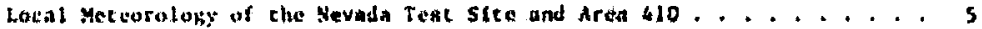

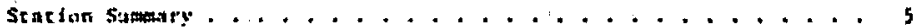

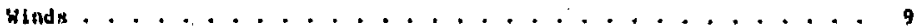

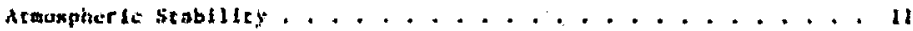

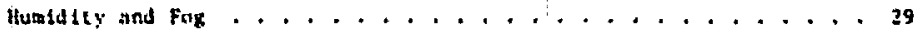

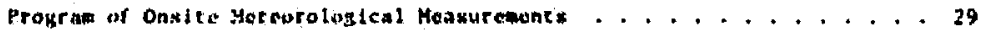

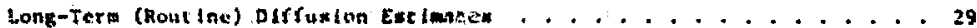

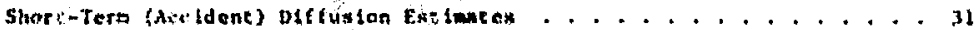

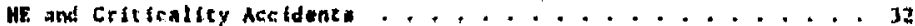

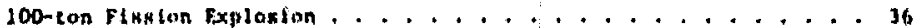

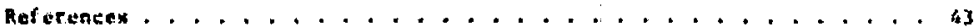




\section{DIFFISION CLIMATOLOGY FOR HYPOTHETICAL ACCIDENTS IN AREA 4LO OF THE NEVADA TEST SITE}

\section{Abstrakt}

the rofingt elfmate around the

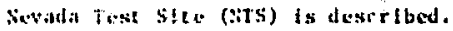

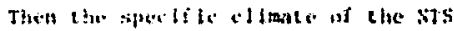

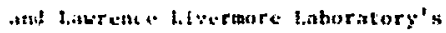

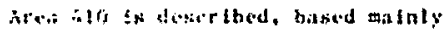

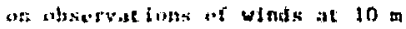

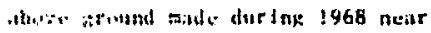

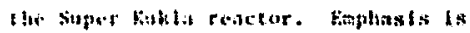

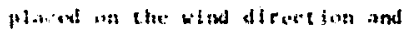

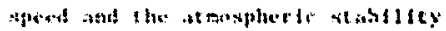

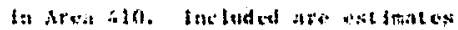

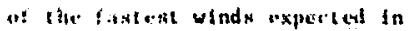

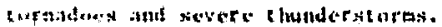

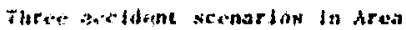

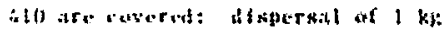

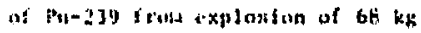
(IST ponteds) al hinth exploxives.

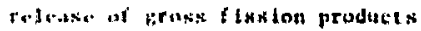
two a $10^{19}$ r biskons aceldent resislt-

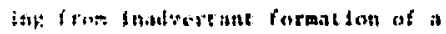

crlefcal wass, athd accldental leconaz lon of $a$ 100-ton fission priary. An Instantaneous Point Source (IPS) cod wis developed at Laurence t.ivermorc Linhorntory and is explatined. The I's code estimites concentrations in the surfite atr of radioactive pirticles that have negligible sclting rotes (have a radfus less than 5 : (a). For each accident, chis code calculated and plocted cor: torar milgs bliat show the cesclatated expokures of the area to radionctive parzicles irom the explosion. The code ralculaced two types of exposure mips: ane: hissed on aritimetically ayoraned, letestated exposures and the of her bitsed on exposure 1 imits chat would be excecded 5 t of the I Ine.

\section{Introduction}

Thin repare describex blo rlsmanlegy of lie Nevada Tent stee atil tofres tharmillzed, Integrated whiteilsen of surface ilf concentra-

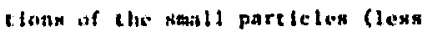
llan 5-gin tadluk) that whe he relcanded the atmospliota by the throc more probable aceldent senarlon at Area 40 , Nevida Teat
Slle, Thls irea, seiffed by l.t.l. personne?, coneatis the Laboracory's лжsembly bullating for nuclenr dovices that ara developed in l.tvermore and sent to Area 410 prior to testing. This document is an adaptation ot a memorancium prepired hy the author for uke in formulat ing the meteorologital portion of a Safety 
Analysis Report (SAR) on Area 410 .

The purpose of this document is twofold: (1) to be included as an append $I x$ to the SAK, and (2) tos serve as an informat Ive document to atmospherlc-diffusion meteorologists and others who may not wish to receive the entire SAR.

\section{Refional Meteorology Around the Nevada Test Site}

GENERAL CLIMATE

Nrs is located in the southern Nevada Jesert. This region is one of the most arid in the entire lindted Statos. Figure I shows the average annual rainiall for vTS and surrounding areas. Flgure $2^{1}$ sitows the average monthly precipitation for selected areas near NTS. The 1962 to 1971 monthly averages and extremes of pre-ipifation for Yucea flat at NT", $18 \mathrm{~km}$ NF of Arna 410, are shown In Fig. 3. The annual surface-water ovaporation rate is many times greater than the rainfall in the reylon. No large bodies of surface watei exist over the ent lre regton, with the axteption of Lake Mead, a man-made reservolr.

in the winter, the primary cause of the arid nature of the region is the so-called "rain shadow" caused by the Sterra Nevada thountains. which make the eastward-moving Pacific storms lous their molsture on the upwind, waetern slopes. Low-presenure centers genorilly approach from the nouchwest to narthwet, usually pasalng to the north of NTS. On the average, etord systan passos ibout once every 7 to 10 days during the winter.

In the summer, Pacific storms do not affect southern Nevada, but moliture sometimes comes from the Gulf of Mextco or the (iulf of California. This moisture falls primarily in isolated convective storms, which can be intense over a few square ktlometres. There may be Jarge variations in precipteation within the storm's area.

The extent of summer showers over couthern Nevada depends primarily on the penetration of the south winds. Thus, precipitation cends to diminish to the north. On rare occasions, tropfial storms occurring of $f$ the west coast of Mextco in late sumer and fill will move northwnrd, bringlng lteavy and widespread precipitat fon to southern Nevada,

SEVERE WEATHER

Thunderstorms and dust dev11s are comon occurrences at the Nevada Teat site. Tornadoes have never been obaerved at MTS, but a feu have ocruryed within $250 \mathrm{~km}$. McDonald. 


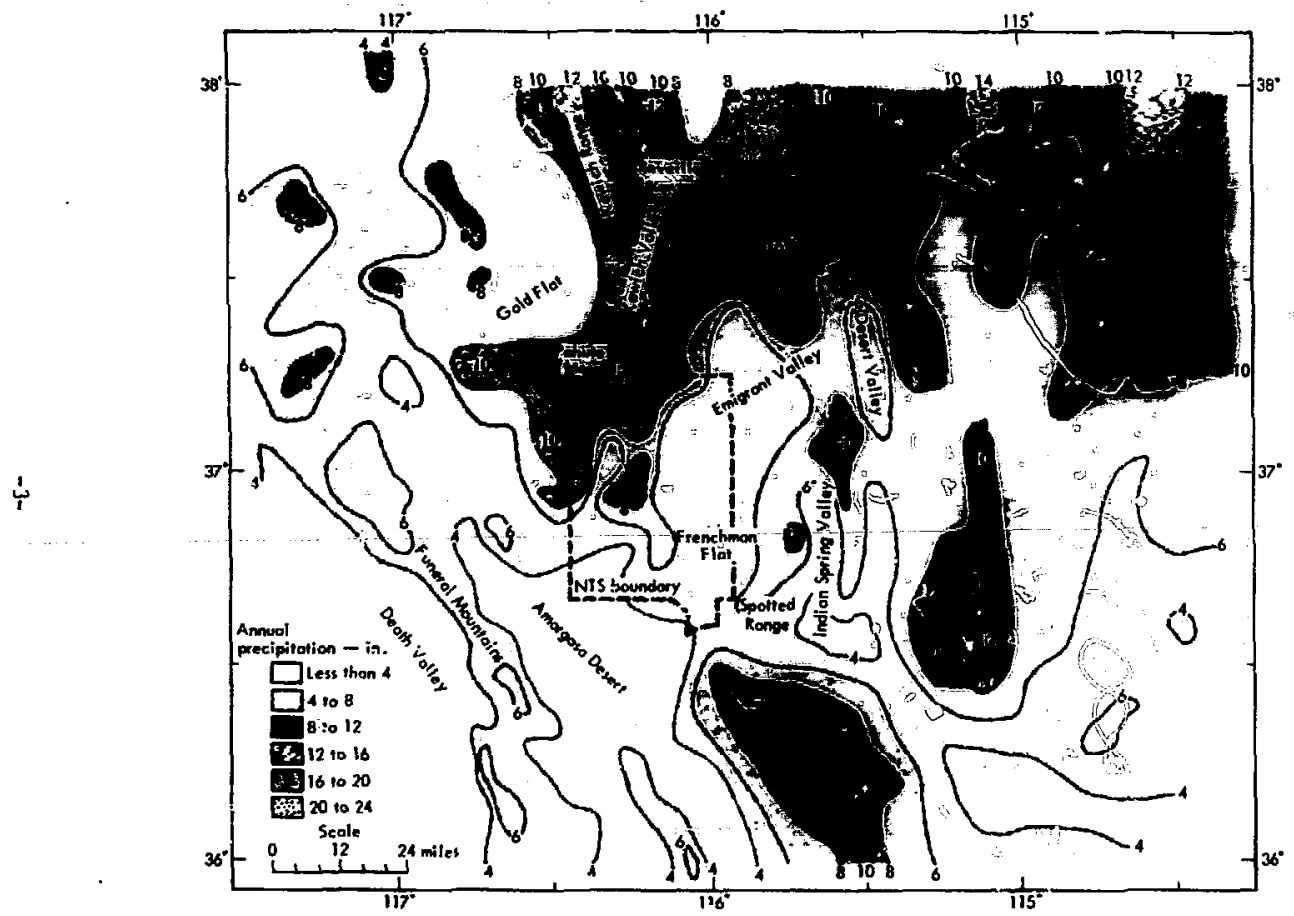

Fig. 1. Mean annual precipttation of Nevada Test Site and vicinity. Bazed on a map by Ralph F. Quiring, Air Resources Laboratory, ias Vegas, Nevada (1965). 


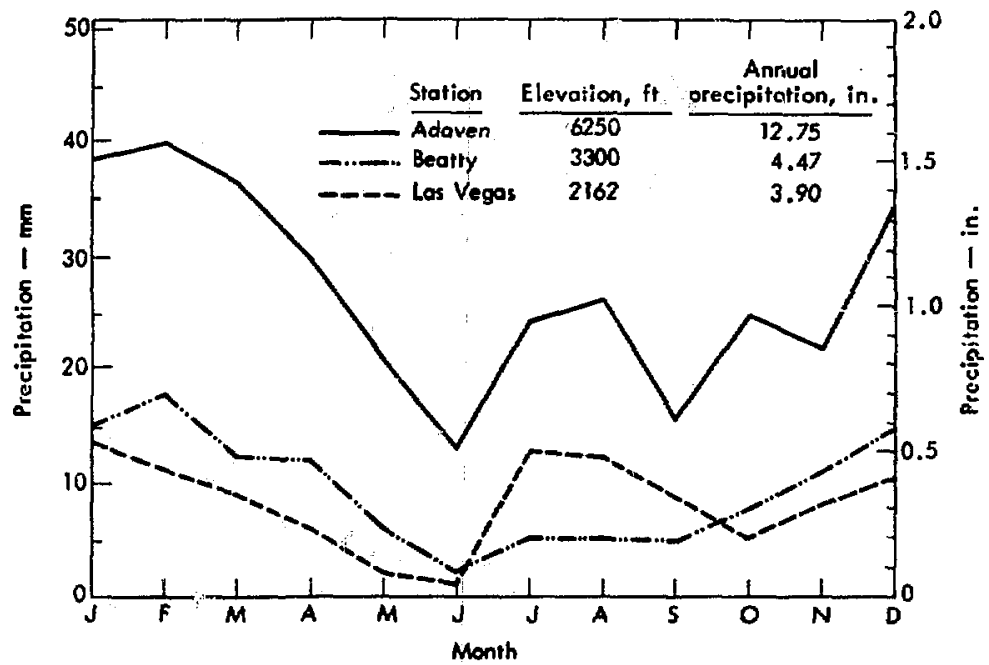

Fig. 2. Normal monthly precipication in millimetres and fuches at several polnts near NTS (1931-1960).

Minor, and Mehte ${ }^{2}$ heve used comonly accepted statiatical techniques tu estimate extrene winds from severe weather conditions. They suggest a maximuardesign wind apeed for NTS from tornadoes of $28 \mathrm{~m} / \mathrm{s}(63 \mathrm{mph})$, with a recurrence Interval of $10^{6}$ years. Their extreme "straight IIne" design wiad epeed -- occurring in other wateorological phenomens, such as thunderetorm - $1894 \mathrm{~m} / \mathrm{s}(210$ mph) for the came recurrence Interval.
AIR-POLLUTION POTENTINL, AT NTS

A study of ritmospherle sabulity conditions in the region ind teates conditions are stable very rately (less tian $5 \%$ of the cime, on an annual basis). Therefore, any locally generated pollution wlll be readily ixixed with the ambient alr, resulting in lower pollutint concentrations at the site's boundary than would occur in most other locations within the United States. 


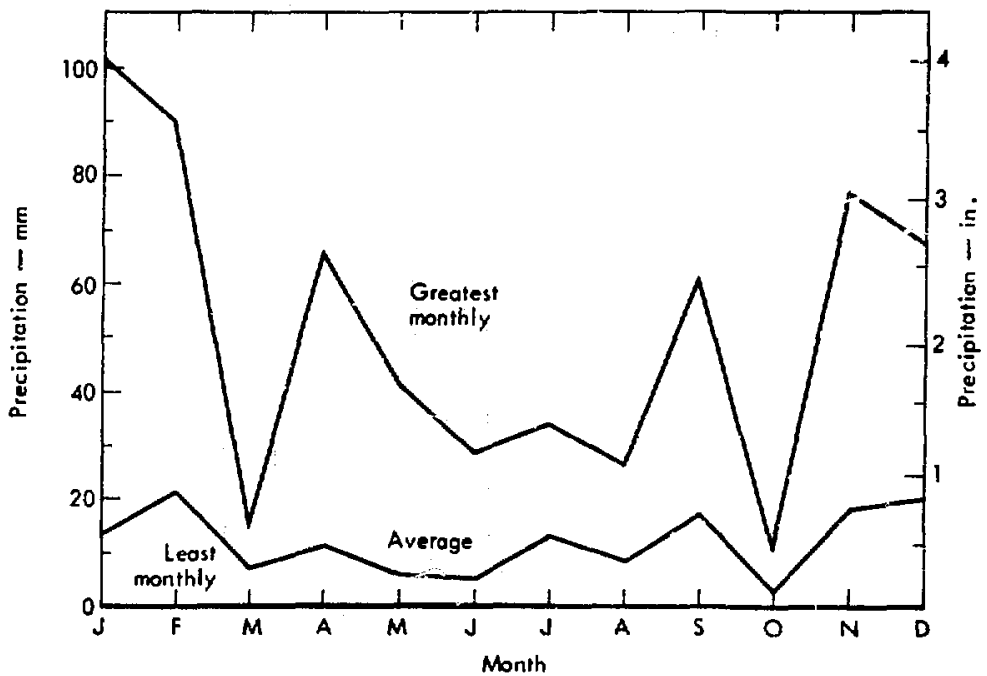

Fig. 3. Precipitation at vucca Flat in NTS, 1962 $\cdots 11$. Least monthly precipitation was less than $0.51 \mathrm{~mm}(0.02 \mathrm{in.})$ for all months.

\section{Local Meteorology of the Nevada Test Site and Area 410}

STATJON SUMOAFi

The tilimate of Area 410 is etsentially the same as that of surrounding reglons. Table 1 gives the: wother summiry for the years 1962 through 1971 for the Yucca Wather station at NTS. Figure 3 shows the average, greatest, and least monthly precipttation at Yucea Flat during the same 10-year pertod, Yucca is the only continuously manned weatiner station that has been in operation at NTS for 10 years. The data reported in Table 1 are fatrly typical of NTS averages, alchough differences arise due to local topography.

The only significant difference between the weather at Area 410 and that of surrounding area is the greater rainfall due to elevarion. 
Table 1. Climatological sumary (1962-1971) of Yucca Flet, Nevada - Nevada Test Slte (latitude 3657'N, longltude $116^{\circ} 03^{\prime} \mathrm{H}$, elevation $3,924 \mathrm{ft}$ ). Data from Natjonal climatic Center, Ashevilie, North Carolina.

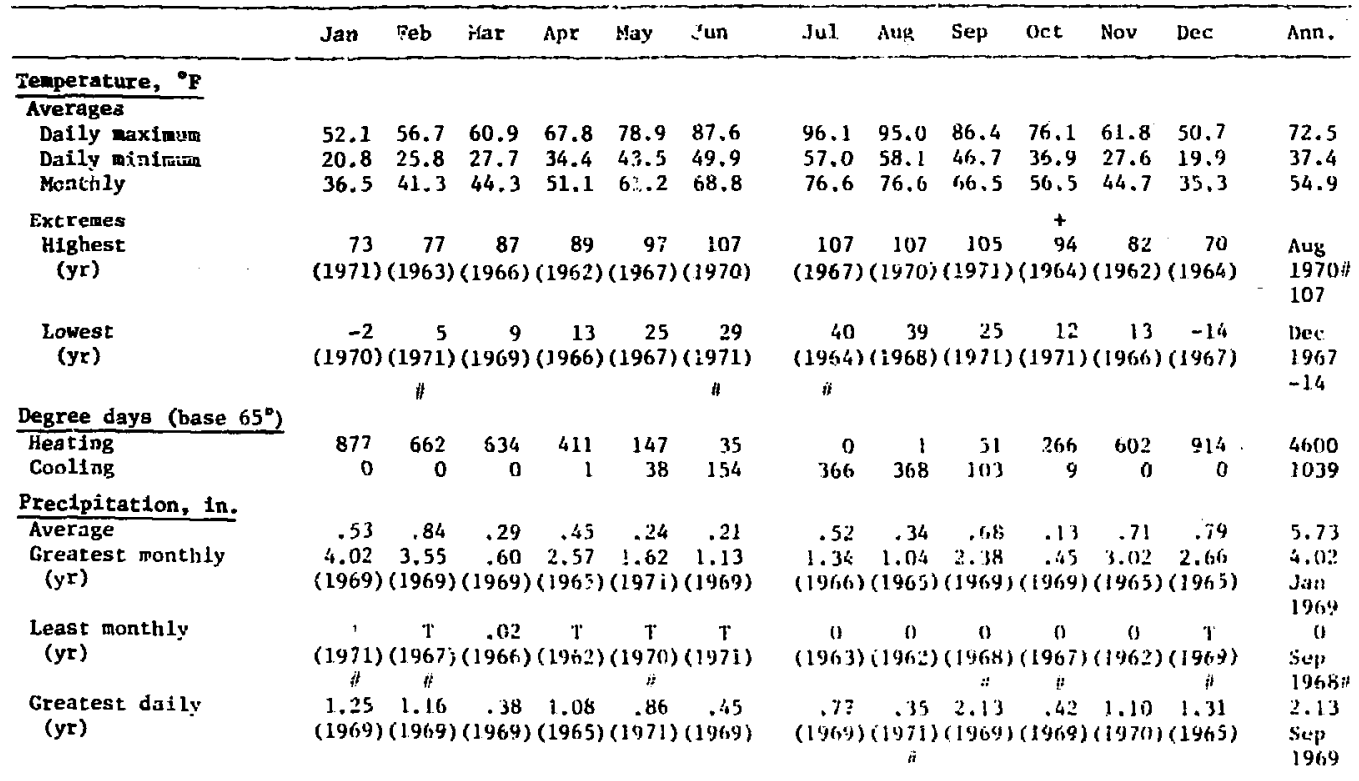


Table 1. coandined.

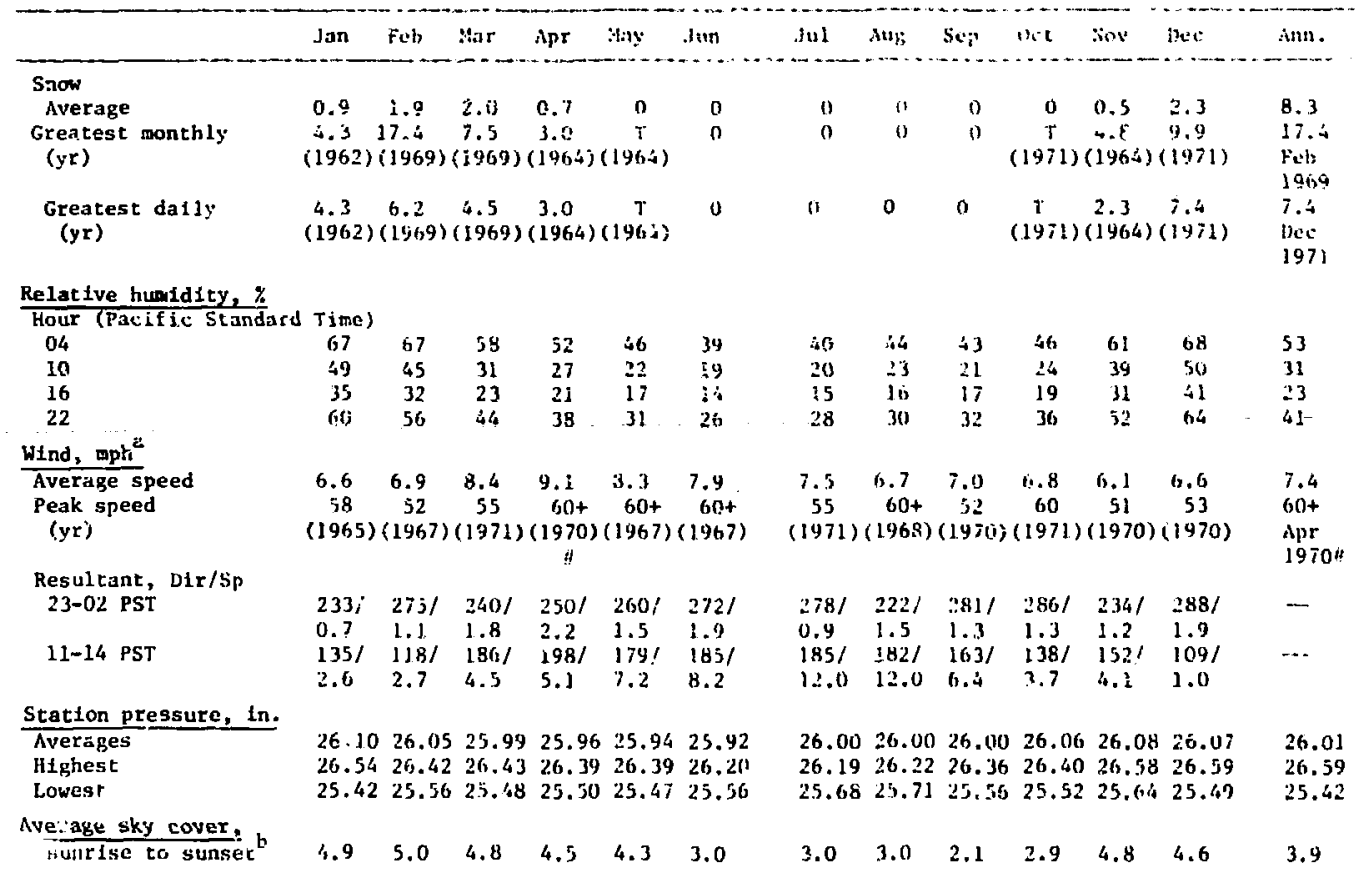


Table 1, continued.

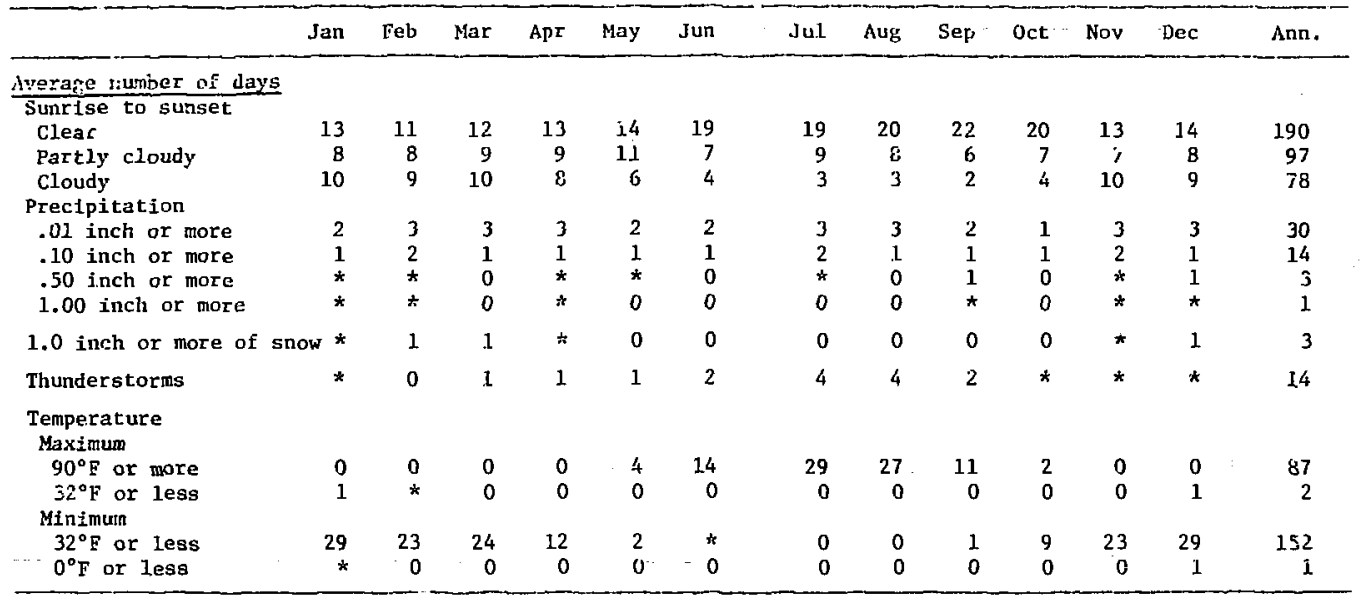

* One or more accurrences during the period of record but average less than 0.5 day.

| Most recent of multiple occurrences,

+ Peak speed exceeded the upper limtt of the analog recorder.

$T$ Trace, an amount too small to measure.

(a) Average and peak speed are for the period starting with lecember 1964. The direction and magnitude of the resultant wind afe from a sumary covering the period December 3964 through May 1969.

(b) Sky cover is expressed in the range from 0 for no clouds to 10 when the sky is completely covered with clouds. Clear, partly cloudy and cloudy are defined as average daytime cloudiness of $0-3,4-7$ and 8-10 tenths, respectively. 
Figure $4^{2}$ shows the way in which

rainfall increases with clevation for

the region around NTS. Dat.a collected

from stations at NTS agree quite.

closely with the plot of average

behavior shown in Fig. 4. Figure $5^{3}$

shows the predicted rainfall for

storms of return periods vaiying

from 1.1 to 1000 years. These

clirves are based on extreme value

theory; the data represent average

behavior for the NTS area.
WINDS

Table $2^{3}$ is a summary of wind data collected at Area 410 during 1964-1968. The recording equipment was unable to measure speeds in excess of $27 \mathrm{~m} / \mathrm{s}(60 \mathrm{mph})$. However, it should be noted that only two observations of winds greater than $17 \mathrm{~m} / \mathrm{s}$ ( $39 \mathrm{mph}$ ) wrra observed. Table $3^{2}$ shows the maximum wind velocities to be expected in the area of NTS.

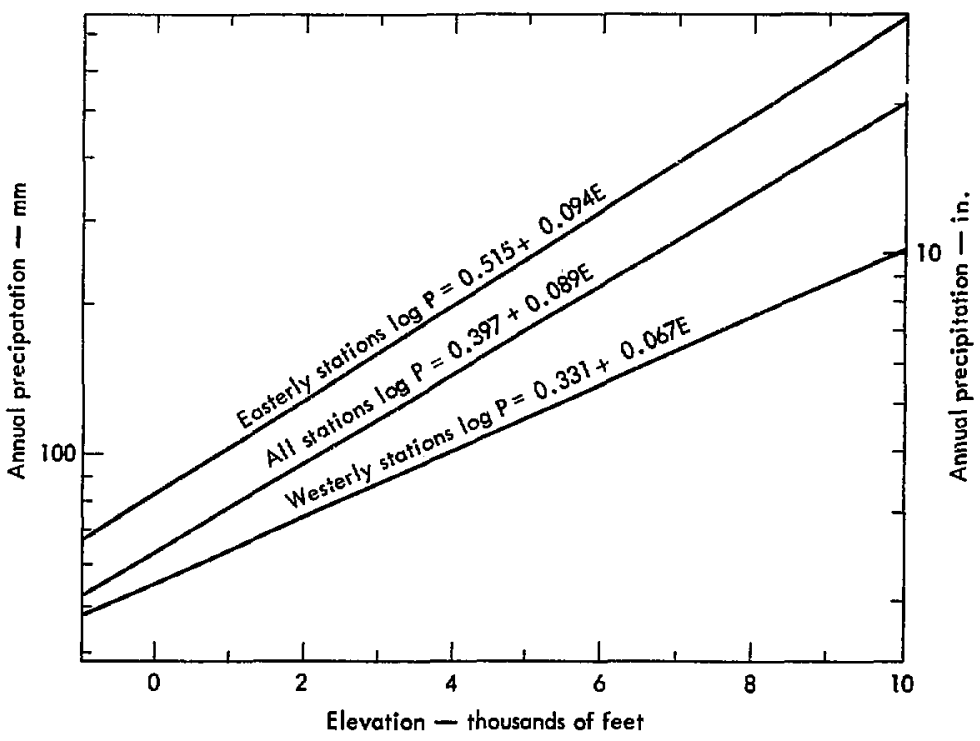

Fig. 4. Log-1inear relationshlp between normal annual precipication ard elevation in southern Nevada (1931-19óo). 


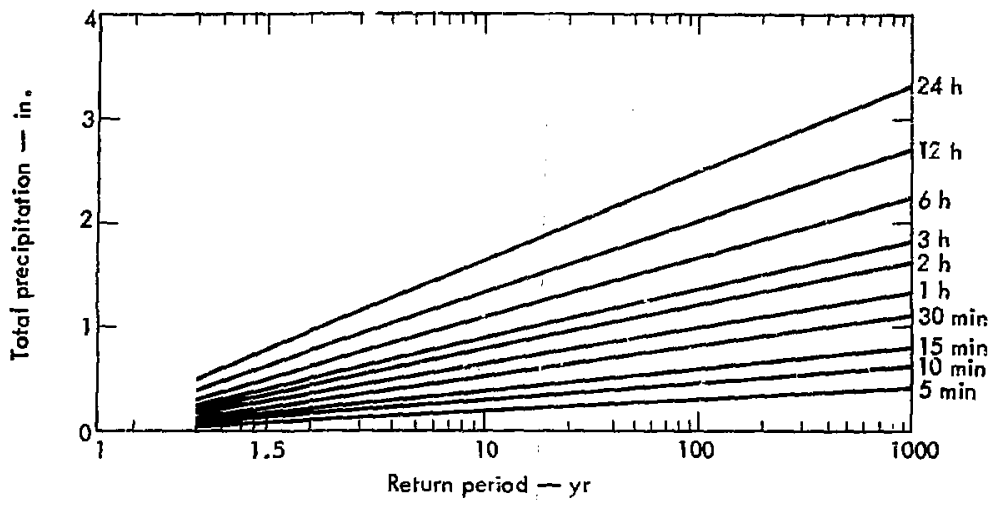

Fig. 5. Probability of precipitation lasting from $5 \mathrm{~min}$ to $24 \mathrm{~h}$ at NTS.

Table 2. Breakdown of wind-speed observations for Area $410,1964-68$ at $9.1 \mathrm{~m}$ above ground. Data from Ref. 3.

\begin{tabular}{ccc}
\hline$\pi / s$ & $1-4$ & Percent of observations \\
\hline $0.5-2.0$ & $5-9$ & 16.8 \\
$2.1-4.2$ & $10-14$ & 44.5 \\
$4.3-6.5$ & $15-19$ & 24.8 \\
$6.6-8.7$ & $20-29$ & 9.3 \\
$8.8-13.2$ & $30-39$ & 4.1 \\
$13.3-17.7$ & $40-49$ & 0.4 \\
$17.8-22.1$ & 250 & $<0.01^{\mathrm{a}}$ \\
222.2 & & $<0.01^{\mathrm{b}}$ \\
\hline
\end{tabular}

a Three observations.

bone observation. 
Table 3. Extreme winds expected at NTS at $9.1 \mathrm{~m}$ (30 ft) above surface.

\begin{tabular}{|c|c|c|c|c|}
\hline \multirow{2}{*}{$\begin{array}{l}\text { Return } \\
\text { perlod, yr }\end{array}$} & \multicolumn{2}{|c|}{ Fastest $^{2}$} & \multicolumn{2}{|c|}{ Gust } \\
\hline & mph & $\mathrm{m} / \mathrm{sec}$ & $\mathrm{mph}$ & $\mathrm{m}_{i}^{\prime} \mathrm{s}$ \\
\hline 2 & 48 & 21 & 62 & 28 \\
\hline 5 & 55 & 25 & 72 & 32 \\
\hline 10 & 61 & 27 & 79 & 35 \\
\hline 50 & 75 & 34 & 97 & 43 \\
\hline 100 & 82 & 37 & 107 & 48 \\
\hline
\end{tabular}

approximately $1-m i n$ average wind.

Wind directions and speeds, as well as stability categories were averaged over half-hour intervals for the entire period from January through December, 1968. The measurements were taken near the Super Kukla reactor bullding at $10 \mathrm{~m}$ above the ground. To study seasonal effects, these data were divided Into winter (Uctober through February) and sumner (March throush September) seasons *

The percentage frequencles of wind directions and average speeds are given In Table 4. In general, the strongest winds blow toward the north through east; the weakest winds blow toward the west through north. The winter and summer winds are remarkably similar in speed and average about $4 \mathrm{~m} / \mathrm{s}$ for all directions.

\#Th1s breakdown of seasons was based on the afternoon resultant wind speeds shown in Table 1.
The frequencies vary more with direction than do the speeds. The annual frequencies show a double maximum -- in the directions toward north through east and toward south through west. The seasonal frequencles show a sir.jle maximum -in the directions toward south through west for winter, and coward north through east for summer.

\section{ATMOSPEERIC STABILITY}

Atmospheric stability was estimated from the wind-direction traces made during 1968, The method outlined by Slade 4 was used to decer-mine these (Pasquill-Gifford) stabllities. A description of these stablitty categorfes is given in Table 5. Table 6 shows the annual percentege frequencles and those for both seasons. The outstanding feature of this table is the relatively 
Table 4. Erequencies and average speeds vs dfrection for inds in Area 410 , 1968 data.

\begin{tabular}{|c|c|c|c|c|c|c|}
\hline \multirow{2}{*}{$\begin{array}{l}\text { Direction } \\
\text { toward which } \\
\text { wind blows }\end{array}$} & \multicolumn{2}{|c|}{ Annual } & \multicolumn{2}{|c|}{ Winter } & \multicolumn{2}{|c|}{ Summer } \\
\hline & $\underset{\%}{\text { Frequency, }}$ & $\begin{array}{l}\text { Av Speed, } \\
\mathrm{m} / \mathrm{s}\end{array}$ & $\underset{\%}{\text { Frequency, }}$ & $\begin{array}{c}\text { Av Speed, } \\
\mathrm{m} / \mathrm{s}\end{array}$ & Frequency, & $\begin{array}{c}\text { Av Speed, } \\
\mathrm{m} / \mathrm{s}\end{array}$ \\
\hline $\mathrm{N}$ & 9.7 & 5.07 & 6.0 & 4.58 & 12.3 & 5.24 \\
\hline NNE & 12.4 & 4.90 & 6.0 & 4.76 & 16.9 & 4.94 \\
\hline NE & 8.9 & 4.25 & $5: 4$ & 4.90 & 11.4 & 4.03 \\
\hline ENE & 10.3 & 3.76 & 6.9 & 5.16 & 12.7 & 3.22 \\
\hline E & 5.7 & 3.11 & 3.3 & 3.96 & 7.3 & 2.84 \\
\hline ESE & 3.6 & 3.28 & $2: 9$ & 3.72 & 4.1 & 3.07 \\
\hline $\mathrm{SE}$ & 2.9 & 2.95 & 3.1 & 3.55 & 2.8 & 2.50 \\
\hline SSE & 4.6 & 3.43 & 5.5 & 3.96 & 4.0 & 2.91 \\
\hline $\mathrm{s}$ & 9.2 & 4.11 & 10.4 & 3.97 & 8.3 & 4.23 \\
\hline SSW & 11.2 & 3.78 & $15: 2$ & 3.54 & 8.4 & 4.09 \\
\hline SW & 10.5 & 3.61 & 18.0 & 3.83 & 5.2 & 3.08 \\
\hline WSW & 5.0 & 4.15 & 10.3 & 4.46 & 1.3 & 2.40 \\
\hline $\mathfrak{W}$ & 0.8 & 5.10 & 2.0 & 5.14 & 0.1 & 1.90 \\
\hline WNW & 0.4 & 2.64 & 0.4 & 2.50 & 0.4 & 2.73 \\
\hline $\mathrm{NW}$ & 1.5 & 2.77 & 1.6 & 2.98 & 1.5 & 2.61 \\
\hline NNW & 3.3 & 3.27 & 3.2 & 3.44 & 3.3 & 3.15 \\
\hline
\end{tabular}

Al1

$\begin{array}{lllllll}\text { directions } & 100.0 & 3.99 & 100.0 & 4.11 & 100.0 & 3.91\end{array}$

low frequency of stable conditions (E and F).* There appears to be no algnificant seasonal variation. Finally, the ungtable categorles (A, B and C) account for about three-

*0ther researchers, e.g., J. B. Krox (in a personal communication), have noted a tendency for a systematic bias, favoring more unstable categories, when the $\Delta \theta$-method is used with atrip charts of wind direction. fourths of all observations. This is a desirable feature since an unstable atmosphere will $\mathrm{mix}$ and dilute ef liuents more capldly than at a site where neutral (D) and stable ( $E$ and $E$ ) categories predominatu. Tabular wind roses, classed by stability category, wind direction, and wind-speed range are given in Tables 7, 8, and 9 for annual. winter, and summer piariods, 
respectively. Note that the total of directiomal and wind-speed frequencies for a given stablitty catchory is the percentage of time that particular stabil1ty occurred; these totals agree with the values

Table 5. Description of atmospher1c stability categories (Pasquil1-Gifford).

\begin{tabular}{|c|c|c|c|}
\hline Category & $\begin{array}{l}\text { Degree of } \\
\text { stability }\end{array}$ & $\begin{array}{l}\text { Angular spread } \\
\text { of wind direction } \\
\text { (30-min period) }\end{array}$ & Description \\
\hline A & Extremely unstable & $150^{\circ}$ & $\begin{array}{l}\text { Rapid mixing; usualiy } \\
\text { occurs on hot after- } \\
\text { noons. }\end{array}$ \\
\hline $\mathbf{B}$ & $\begin{array}{l}\text { Moderately } \\
\text { unstable }\end{array}$ & $120^{\circ}$ & $\begin{array}{l}\text { Moderate mixing; } \\
\text { usually occurs on warm } \\
\text { days. }\end{array}$ \\
\hline c & S1 ight ly unstable & $90^{\circ}$ & $\begin{array}{l}\text { Usually occurs in } \\
\text { daytime. }\end{array}$ \\
\hline D & Neutral stability & $60^{\circ}$ & $\begin{array}{l}\text { Occurs day or night, } \\
\text { of ten during overcast } \\
\text { skies. }\end{array}$ \\
\hline $\mathfrak{E}$ & Sightsy stable & $30^{\circ}$ & $\begin{array}{l}\text { Slow dilution; can } \\
\text { occur day or night. }\end{array}$ \\
\hline $\mathbf{F}$ & Moderately stable & $15^{\circ}$ & $\begin{array}{l}\text { Very slow dilution; } \\
\text { occurs at night with } \\
\text { clear skies. }\end{array}$ \\
\hline
\end{tabular}

Tible 6. Percentage frequency of stabllity categories it Area 410.

\begin{tabular}{|c|c|c|c|c|}
\hline \multirow[b]{2}{*}{ stability catchory } & \multicolumn{4}{|c|}{ Frequency, $z$} \\
\hline & Annual & Hinter (act-Feb) & Sumer & (Mar-Sepr) \\
\hline$\dot{n}$ & 27.5 & 29.6 & & 26.1 \\
\hline$\|$ & 17.1 & 15.6 & & 18.2 \\
\hline c: & 26.9 & 28.2 & & 26.3 \\
\hline $\mathbf{n}$ & 23.7 & 21.6 & & 25.2 \\
\hline$r$ & 4.3 & 6.4 & & 4.2 \\
\hline$r$ & 0.3 & 0.6 & & 0.2 \\
\hline
\end{tabular}


Table 7. Annual frequencies of winds.

\begin{tabular}{|c|c|c|c|c|c|c|c|c|}
\hline \multirow{2}{*}{$\begin{array}{l}\text { Direction toward } \\
\text { which the } \\
\text { wind blows }\end{array}$} & \multicolumn{8}{|c|}{ Frequency, $x$} \\
\hline & $0.5-2.0$ & $2.1-3.5$ & $\begin{array}{c}\text { winds having } \\
3.6-5.5\end{array}$ & $\begin{array}{c}\text { speeds (in } \\
5.6-9.0\end{array}$ & $\begin{array}{l}\mathrm{m} / \mathrm{s}) \text { of : } \\
9.1-12.0\end{array}$ & $>12$ & $\begin{array}{l}\text { Totals by } \\
\text { ditect ion }\end{array}$ & $\begin{array}{l}\text { Av } \\
\text { speed }\end{array}$ \\
\hline \multicolumn{9}{|c|}{ stabllity Category $A$} \\
\hline $\mathbf{N}$ & 0.47 & 0.98 & 0.61 & 0.17 & 0.0 & 0.0 & 2.23 & 3.30 \\
\hline NE & 0.48 & 0.67 & 0.84 & 0.26 & 0.0 & 0.0 & 2.25 & 3.64 \\
\hline ENE & 0.44 & 0.41 & 0.57 & 0.47 & 0.14 & 0.01 & 2.04 & 4.58 \\
\hline $\mathbf{E}$ & 0.35 & 0.53 & 0.38 & 0.15 & 0.0 & 0.0 & 1.41 & 3.37 \\
\hline ESE & 0.51 & 0.33 & 0.39 & 0.14 & 0.0 & 0.0 & 1.37 & 3.18 \\
\hline $\mathbf{S E}$ & 0.74 & 0.35 & 0.31 & 0.16 & 0.0 & 0.0 & 1.56 & 2.87 \\
\hline SSW & 0.50 & 0.75 & 0.73 & 0.60 & 0.01 & 0.0 & 2.59 & 4.07 \\
\hline SW & 0.38 & 1.0 & 0.88 & 0.70 & 0.09 & 0.01 & 3.06 & 4.41 \\
\hline WSW & 0.25 & 0.99 & 0.94 & 0.53 & 0.16 & 0.05 & 2.81 & 4.75 \\
\hline W & 0.02 & 0.19 & 0.33 & 0.19 & 0.06 & 0.0 & 0.78 & 5.19 \\
\hline WNW & 0.02 & 0.02 & 0.0 & 0.0 & 0.0 & 0.0 & 0.04 & 2.03 \\
\hline NW & 0.07 & 0.09 & 0.07 & 0.0 & 0.0 & 0.0 & 0.23 & 2.86 \\
\hline NNW & 0.25 & 0.49 & 0.12 & 0.03 & 0.0 & 0.0 & 0.89 & 2.75 \\
\hline Total & 6.08 & 8.19 & 8.25 & 4.42 & 0.53 & 0.07 & 27.54 & 3.88 \\
\hline
\end{tabular}


Table 7, continued.

Stability Category B

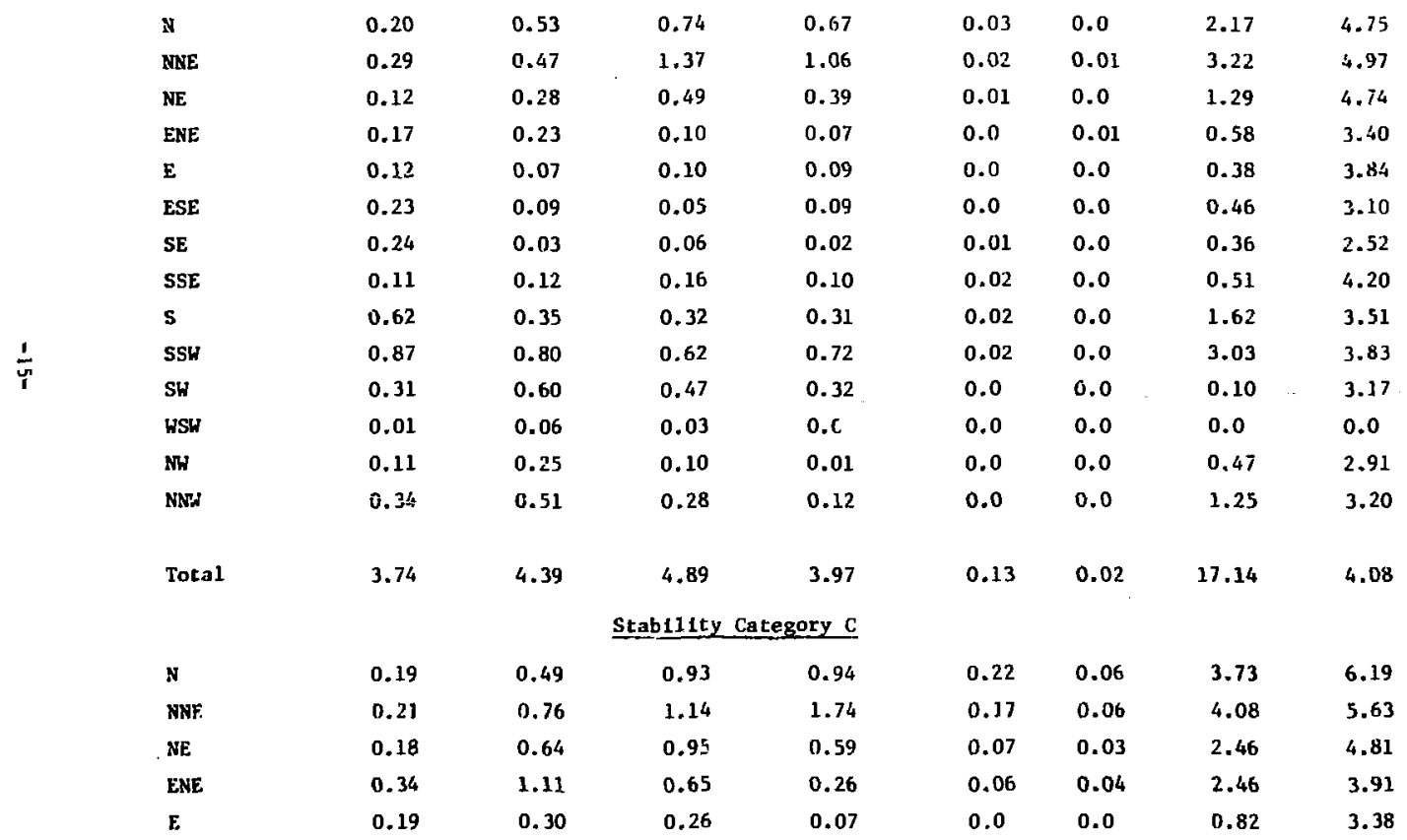


Table 7, continued.

\begin{tabular}{|c|c|c|c|c|c|c|c|c|}
\hline ESE & 0.19 & 0.21 & 0.14 & 0.06 & 0.01 & 0.0 & 0.61 & 3.29 \\
\hline SE & 0.23 & 0.05 & 0.13 & 0.05 & 0.02 & 0.0 & 0,48 & 3.32 \\
\hline SSE & 0.44 & 0.17 & 0.24 & 0.11 & 0.02 & 0.0 & 0.98 & 3.20 \\
\hline$s$ & 0.52 & 0.52 & 0.82 & 0.60 & 0.09 & 0.0 & 2.55 & 4.38 \\
\hline SSW & 0.93 & 0.87 & 0.75 & 0.55 & 0.15 & 0.0 & 3.25 & 3.88 \\
\hline SW & 0.98 & 1.20 & 0.77 & 0.35 & 0.05 & 0.0 & 3.35 & 3.33 \\
\hline USW & 0.14 & 0.24 & 0.10 & 0.09 & 0.0 & 0.0 & 0.57 & 3.44 \\
\hline $\mathbf{H}$ & 0.0 & 0.0 & 0.0 & 0.0 & 0.0 & 0.0 & 0.0 & 0.0 \\
\hline $\operatorname{thax}$ & 0.03 & 0.06 & 0.0 & 0.0 & 0.0 & 0.0 & 0.09 & 2.28 \\
\hline$M$ & 0.22 & 0.23 & 0.10 & 0.01 & 0.0 & 0.0 & 0.56 & 2.58 \\
\hline $\mathrm{mw}$ & 0.19 & 0.28 & 0.25 & 0.18 & 0.02 & 0.0 & 0.92 & 4.00 \\
\hline Total & 4.88 & 7.13 & 7.23 & 6.60 & 0.88 & 0.19 & 26.91 & 4.43 \\
\hline \multicolumn{9}{|c|}{ Stability Category D } \\
\hline $\mathbf{N}$ & 0.04 & 0.11 & 0.32 & 0.66 & 0.24 & 0.02 & 1.39 & 6.81 \\
\hline MNE & 0.14 & 0.47 & 0.64 & 0.58 & 0.10 & 0.11 & 2.04 & 5.56 \\
\hline NE & 0.26 & 1.01 & 0.90 & 0.54 & 0.07 & 0.03 & 2.81 & 4.41 \\
\hline ENE & 0.64 & 2.48 & 1.31 & 0.35 & 0.01 & 0.04 & 4.83 & 3.51 \\
\hline$E$ & 0.53 & 1.04 & 0.25 & 0.13 & 0.05 & 0.0 & 2.00 & 3.09 \\
\hline ESE & 0.14 & 0.32 & 0.24 & 0.13 & 0.03 & 0.0 & 0.86 & 3.99 \\
\hline SE & 0.11 & 0.12 & 0.11 & 0.06 & 0.0 & 0.0 & 0.40 & 3.53 \\
\hline SSE & 0.36 & 0.36 & 0.35 & 0.18 & 0.01 & 0.0 & 1.26 & 3.55 \\
\hline $\mathbf{s}$ & 0.24 & 0.55 & 0.83 & 0.85 & 0.09 & 0.0 & 2.56 & 4.99 \\
\hline
\end{tabular}


Table 7, continued.

\begin{tabular}{lllllllll}
\hline SSW & 0.58 & 0.51 & 0.22 & 0.43 & 0.06 & 0.0 & 1.80 & 3.85 \\
SW & 0.65 & 0.77 & 0.25 & 0.19 & 0.05 & 0.01 & 1.92 & 3.21 \\
WSW & 0.21 & 0.57 & 0.20 & 0.21 & 0.0 & 0.01 & 1.20 & 3.71 \\
W & 0.0 & 0.01 & 0.0 & 0.0 & 0.0 & 0.0 & 0.01 & 2.80 \\
WNW & 0.06 & 0.06 & 0.05 & 0.03 & 0.0 & 0.0 & 0.20 & 3.45 \\
NW & 0.07 & 0.11 & 0.03 & 0.04 & 0.0 & 0.0 & 0.25 & 3.30 \\
WWW & 0.04 & 0.03 & 0.05 & 0.09 & 0.0 & 0.0 & 0.21 & 4.85 \\
Total & & & & & & & & \\
& 4.07 & 8.52 & 5.75 & 4.47 & 0.71 & 0.22 & 23.74 & 4.15 \\
N & & & Stabiltty categoryE & & & & \\
NNE & 0.01 & 0.0 & 0.02 & 0.02 & 0.0 & 0.0 & 0.05 & 4.99 \\
NE & 0.0 & 0.03 & 0.0 & 0.0 & 0.0 & 0.0 & 0.03 & 2.80 \\
ENE & 0.01 & 0.02 & 0.05 & 0.0 & 0.01 & 0.0 & 0.09 & 4.46 \\
E & 0.09 & 0.30 & 0.01 & 0.01 & 0.0 & 0.0 & 0.31 & 2.55 \\
ESE & 0.20 & 0.71 & 0.11 & 0.03 & 0.0 & 0.0 & 1.05 & 2.82 \\
SE & 0.01 & 0.19 & 0.04 & 0.03 & 0.0 & 0.0 & 0.27 & 3.50 \\
SSE & 0.03 & 0.02 & 0.05 & 0.01 & 0.0 & 0.0 & 0.11 & 3.58 \\
S & 0.02 & 0.14 & 0.14 & 0.06 & 0.0 & 0.0 & 0.36 & 4.14 \\
SSW & 0.12 & 0.18 & 0.25 & 0.11 & 0.0 & 0.0 & 0.66 & 3.93 \\
SW & 0.10 & 0.19 & 0.05 & 0.17 & 0.0 & 0.0 & 0.51 & 4.17 \\
WSW & 0.11 & 0.12 & 0.07 & 0.09 & 0.0 & 0.01 & 0.40 & 4.00 \\
W & 0.03 & 0.13 & 0.09 & 0.09 & 0.0 & 0.0 & 0.34 & 4.32 \\
NW & 0.01 & 0.0 & 0.0 & 0.0 & 0.0 & 0.0 & 0.01 & 1.25
\end{tabular}




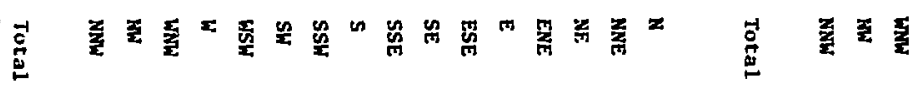

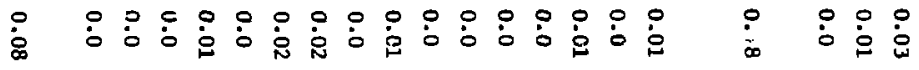

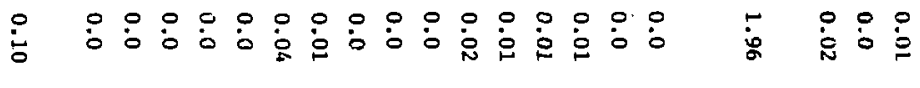

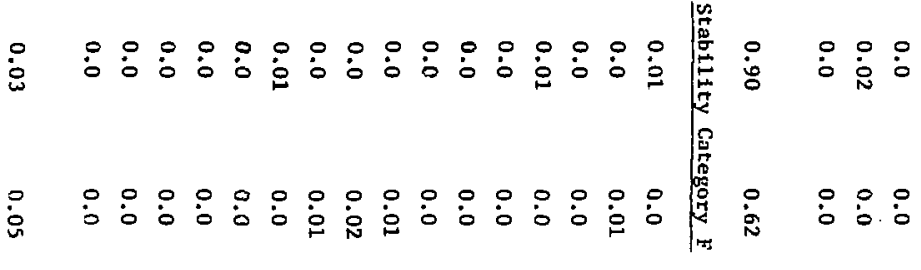

$\dot{0} \dot{0} \dot{0} \dot{0} \dot{0} \dot{0} \dot{0} \dot{0} \dot{0} \dot{0} \dot{0} \dot{0} \dot{0} \dot{0} \dot{0} \dot{0}$

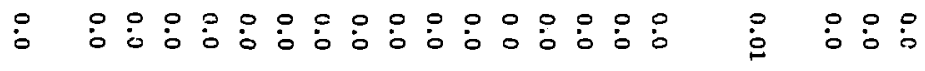

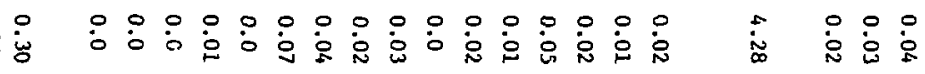

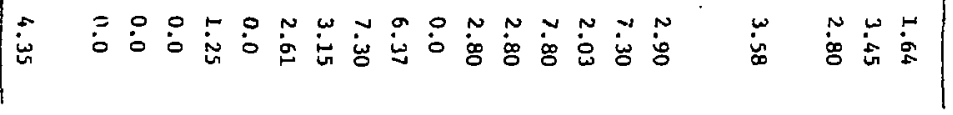


Table 8. Winter (Oct-Feb) frequencles of winds.

\begin{tabular}{|c|c|c|c|c|c|c|c|c|}
\hline \multirow{2}{*}{$\begin{array}{l}\text { Direction toward } \\
\text { which the } \\
\text { wind blows }\end{array}$} & \multicolumn{8}{|c|}{ Frequency, \% } \\
\hline & $0.5-2.0$ & $\begin{array}{r}\text { For } \\
2.1-3.5\end{array}$ & $\begin{array}{c}\text { winds having } \\
3.6-5.5\end{array}$ & $\begin{array}{l}\text { speeds (in } \\
5.6-9.0\end{array}$ & $\begin{array}{l}\mathrm{m} / \mathrm{s}) \text { of : } \\
9.1-12.0\end{array}$ & $>12$ & $\begin{array}{l}\text { Totals by } \\
\text { direction }\end{array}$ & $\begin{array}{l}\text { Av } \\
\text { speed }\end{array}$ \\
\hline \multicolumn{9}{|c|}{ Stability Category $A$} \\
\hline $\mathrm{x}$ & 0.56 & 0.38 & n.10 & 0.20 & 0.0 & 0.0 & 1.24 & 2.97 \\
\hline MNE & 0.43 & 0.38 & 0.20 & 0.18 & 0.03 & 0.0 & 1.22 & 3.40 \\
\hline NE & 0.43 & 0.23 & 0.28 & 0.10 & 0.0 & 0.0 & 1.04 & 3.06 \\
\hline ENE & 0.48 & 0.41 & 0.68 & 0.87 & 0.33 & 0.03 & 2.78 & 5.41 \\
\hline $\mathbf{E}$ & 0.33 & 0.33 & 0.26 & 0.23 & 0.0 & 0.0 & 1.15 & 3.65 \\
\hline ESE & 0.41 & 0.23 & 0.28 & 0.18 & 0.0 & 0.0 & 1.10 & 3.40 \\
\hline SE & 0.66 & 0.26 & 0.33 & 0.26 & 0.0 & 0.0 & 1.51 & 3.28 \\
\hline SSE & 0.69 & 0.26 & 0.46 & 0.20 & 0.08 & 0.0 & 1.69 & 3.54 \\
\hline $\mathbf{s}$ & 0.89 & 0.28 & 0.31 & 0.56 & 0.05 & 0.0 & 2.09 & 3.79 \\
\hline SSW & 0.61 & 0.61 & 0.59 & 0.51 & 0.03 & 0.0 & 2.35 & 3.91 \\
\hline Sw & 0.48 & 1.35 & 1.58 & 1.33 & 0.20 & 0.03 & 4.97 & 4.80 \\
\hline WSW & 0.41 & 1.91 & 2.22 & 1.28 & 0.38 & 0.13 & 6.33 & 4.94 \\
\hline$w$ & 0.05 & 0.45 & 0.79 & 0.46 & 0.15 & 0.0 & 1.91 & 5.18 \\
\hline WNW & 0.0 & 0.0 & 0.0 & 0.0 & 0.0 & 0.0 & 0.0 & 0.0 \\
\hline $\mathrm{sw}$ & 0.0 & 0.0 & 0.0 & 0.0 & 0.0 & 0.0 & 0.0 & 0.0 \\
\hline ANM & 0.15 & 0.05 & 0.03 & 0.03 & 0.0 & 0.0 & 0.26 & 2.63 \\
\hline Total & 6.58 & 7.14 & 8.09 & 6.39 & 1.25 & 0.19 & 29.64 & 4.31 \\
\hline
\end{tabular}


Table 8, continued.

Stability Category B

\begin{tabular}{|c|c|c|c|c|c|c|c|c|}
\hline $\mathbf{N}$ & 0.26 & 0.69 & 0.15 & 0.28 & 0.05 & 0.0 & 1.43 & 3.85 \\
\hline NTE & 0.31 & 0.31 & 0.38 & 0.1 .3 & 0.05 & 0.03 & 1.21 & 4.06 \\
\hline NE & 0.13 & 0.18 & 0.23 & 0.15 & 0.03 & 0.0 & 0.72 & 4.34 \\
\hline ENE & 0.18 & 0.13 & 0.10 & 0.05 & 0.0 & 0.03 & 0.49 & 3.79 \\
\hline $\mathbf{E}$ & 0.05 & 0.08 & 0.18 & 0.13 & 0.0 & 0.0 & 0.44 & 4.67 \\
\hline ESE & 0.23 & 0.15 & 0.03 & 0.10 & 0.0 & 0.0 & 0.51 & 3.09 \\
\hline SE & 0.13 & 0.03 & 0.15 & 0.03 & 0.03 & 0.0 & 0.37 & 3.96 \\
\hline SSE & 0.08 & 0.18 & 0.28 & 0.18 & 0.05 & 0.0 & 0.77 & 4.83 \\
\hline s & 0.97 & 0.43 & 0.36 & 0.36 & 0.05 & 0.0 & 2.17 & 3.32 \\
\hline SSW & 1.35 & 0.94 & 0.61 & 0.99 & 0.05 & 0.0 & 3.94 & 3.77 \\
\hline SW & 0.43 & 0.46 & 0.38 & 0.69 & 0.0 & 0.0 & 1.96 & 4.38 \\
\hline WSh & 0.0 & 0.03 & 0.03 & 0.0 & 0.0 & 0.0 & 0.06 & 3.68 \\
\hline w & 0.0 & 0.0 & 0.0 & 0.0 & 0.0 & 0.0 & 0.0 & 0.0 \\
\hline WWW & 0.0 & 0.0 & 0.0 & 0.0 & 0.0 & 0.0 & 0.0 & 0.0 \\
\hline NW & 0.08 & 0.28 & 0.13 & 0.03 & 0.0 & 0.0 & $0.4^{\circ}=$ & 3.37 \\
\hline NNW & 0.41 & 0.31 & 0.15 & 0.20 & 0.0 & 0.0 & 1.017 & 3.29 \\
\hline Total & 4.61 & 4.10 & 3.16 & 3.32 & 0.31 & 0.06 & 15.56 & 3.86 \\
\hline \multicolumn{9}{|c|}{ Stability Category $C$} \\
\hline N & 0.38 & 0.51 & 0.38 & 0.59 & 0.28 & 0.0 & 2.14 & 5,09 \\
\hline NNE & 0.31 & 0.46 & 0.56 & 0.64 & 0.26 & 0.03 & 2.26 & 5.35 \\
\hline NE & 0.26 & 0.38 & 0.43 & 0.87 & 0.15 & 0.03 & 2.12 & 5.53 \\
\hline
\end{tabular}


Table 8, continued.

\begin{tabular}{|c|c|c|c|c|c|c|c|c|}
\hline ENE & 0.18 & 0.48 & 0.36 & 0.38 & 0.15 & 0.10 & 1.65 & 5.49 \\
\hline $\mathrm{E}$ & 0.13 & 0.18 & 0.26 & 0.05 & 0.0 & 0.0 & 0.62 & 3.57 \\
\hline ESE & 0.05 & 0.15 & 0.05 & 0.13 & 0.03 & 0.0 & 0.41 & 4.82 \\
\hline $\mathrm{SE}$ & 0.18 & 0.10 & 0.20 & 0.03 & 0.05 & 0.0 & 0.56 & 3.86 \\
\hline SSE & 0.36 & 0.28 & 0.38 & 0.15 & 0.05 & 0.0 & 1.22 & 3.76 \\
\hline s & 0.97 & 0.74 & 0.59 & 0.71 & 0.10 & 0.0 & 3.11 & 3.93 \\
\hline SSW & 1.99 & 1.20 & 0.94 & 0.54 & 0.28 & 0.0 & 4.95 & 3.44 \\
\hline SW & 1.71 & 2.04 & 1.35 & 0.79 & 0.13 & 0.0 & 6.02 & 3.51 \\
\hline WSW & 0.18 & 0.31 & 0.20 & 0.20 & 0.0 & 0.0 & 0.89 & 3.89 \\
\hline W & 0.0 & 0.0 & 0.0 & 0.0 & 0.0 & 0.0 & 0.0 & 0.0 \\
\hline WNW & 0.03 & 0.05 & 0.0 & 0.0 & 0.0 & 0.0 & 0.08 & 2.22 \\
\hline $\mathrm{NW}$ & $0.3 \mathrm{~J}$ & 0.20 & 0.13 & 0.03 & 0.0 & 0.0 & 0.67 & 2.62 \\
\hline NNW & 0.43 & 0.36 & 0.36 & 0.31 & 0.03 & 0.0 & 1.49 & 3.87 . \\
\hline Total & 7.47 & 7.44 & 6.19 & 5.42 & 1.51 & 0.16 & 28.19 & 4.32 \\
\hline \multicolumn{9}{|c|}{ Stability Catagory D } \\
\hline $\mathbf{N}$ & 0.10 & 0.05 & 0.18 & 0.43 & 0.33 & 0.0 & 1.09 & 7.07 \\
\hline NNE & 0.15 & 0.18 & 0.26 & 0.54 & 0.18 & 0.03 & 1.34 & 6.09 \\
\hline $\mathrm{NE}$ & 0.13 & 0.20 & 0.28 & 0.66 & 0.10 & 0.05 & 1.42 & 6.07 \\
\hline ENE & 0.28 & 0.33 & 0.41 & 0.56 & 0.03 & 0.08 & 1.69 & 5.17 \\
\hline E & 0.18 & 0.20 & 0.15 & 0.20 & 0.13 & 0.0 & 0.86 & 5.00 \\
\hline ESE & 0.05 & 0.20 & 0.28 & 0.08 & 0.05 & 0.0 & 0.66 & 4.56 \\
\hline SE & 0.13 & 0.18 & 0.08 & 0.13 & 0.0 & 0.0 & 0.52 & 3.81 \\
\hline
\end{tabular}


Table 8, continued.

\begin{tabular}{lllllllll}
\hline SSE & 0.28 & 0.38 & 0.23 & 0.38 & 0.03 & 0.0 & 1.30 & 4.27 \\
S & 0.36 & 0.48 & 0.61 & 0.89 & 0.13 & 0.0 & 2.47 & 5.04 \\
SSW & 1.12 & 0.77 & 0.31 & 0.69 & 0.10 & 0.0 & 2.99 & 3.70 \\
SW & 1.35 & 1.50 & 0.61 & 0.46 & 0.13 & 0.03 & 4.08 & 3.39 \\
WSW & 0.33 & 0.94 & 0.48 & 0.51 & 0.0 & 0.03 & 2.29 & 4.11 \\
W & 0.0 & 0.03 & 0.0 & 0.0 & 0.0 & 0.0 & 0.03 & 2.80 \\
WNW & 0.03 & 0.08 & 0.10 & 0.0 & 0.0 & 0.0 & 0.21 & 3.41 \\
NW & 0.10 & 0.23 & 0.05 & 0.08 & 0.0 & 0.0 & 0.46 & 3.44 \\
NNW & 0.10 & 0.08 & 0.08 & 0.13 & 0.0 & 0.0 & 0.39 & 4.26 \\
& & & & & & & & \\
TOta1 & 4.69 & 5.83 & 4.11 & 5.74 & 1.21 & 0.22 & 21.80 & 4.53 \\
& & & Stability & CategoryE & & & & 4.28 \\
N & 0.03 & 0.0 & 0.0 & 0.03 & 0.0 & 0.0 & 0.06 & 4.0 \\
NNE & 0.0 & 0.0 & 0.0 & 0.0 & 0.0 & 0.0 & 0.0 & 0.0 \\
NE & 0.03 & 0.0 & 0.0 & 0.0 & 0.03 & 0.0 & 0.06 & 5.90 \\
ENE & 0.05 & 0.03 & 0.03 & 0.03 & 0.0 & 0.0 & 0.14 & 3.59 \\
E & 0.10 & 0.05 & 0.03 & 0.08 & 0.0 & 0.0 & 0.26 & 3.79 \\
ESE & 0.03 & 0.08 & 0.0 & 0.05 & 0.0 & 0.0 & 0.16 & 3.92 \\
SE & 0.03 & 0.0 & 0.08 & 0.03 & 0.0 & 0.0 & 0.14 & 4.43 \\
SSE & 0.03 & 0.10 & 0.18 & 0.15 & 0.0 & 0.0 & 0.46 & 4.85 \\
S & 0.13 & 0.05 & 0.15 & 0.20 & 0.0 & 0.0 & 0.53 & 4.61 \\
SSW & 0.20 & 0.18 & 0.10 & 0.41 & 0.0 & 0.0 & 0.89 & 4.72 \\
SW & 0.18 & 0.23 & 0.15 & 0.20 & 0.0 & 0.03 & 0.79 & 4.38
\end{tabular}




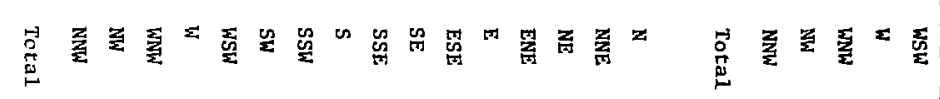

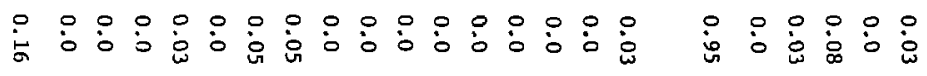

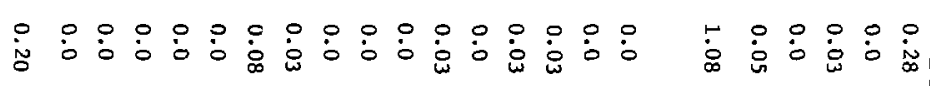

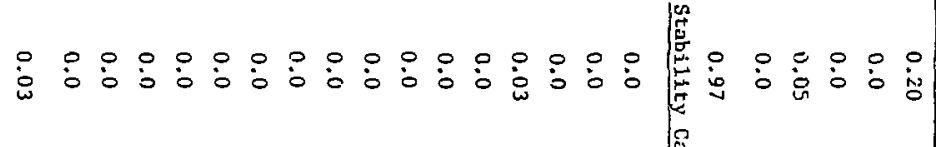

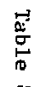

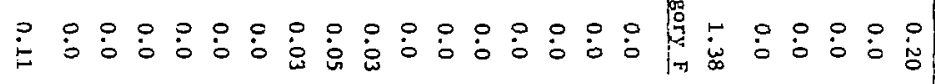

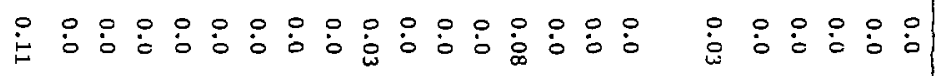

$\dot{0} \dot{0} \dot{0} \dot{0} \dot{0} \dot{0} \dot{0} \dot{0} \dot{0} \dot{0} \dot{0} \dot{0} \dot{0} \dot{0} \dot{0} \dot{0} \dot{0} \dot{0} \dot{0} \dot{0}$

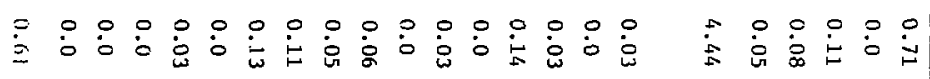

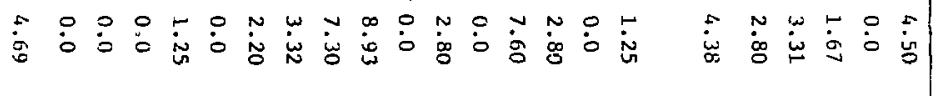


Table 9. Summer (March-September) frequencies of winds.

\begin{tabular}{|c|c|c|c|c|c|c|c|c|}
\hline \multirow{2}{*}{$\begin{array}{l}\text { Direction toward } \\
\text { which the } \\
\text { wind blows }\end{array}$} & \multicolumn{7}{|c|}{ Frequency, $\%$} & \multirow[b]{2}{*}{$\begin{array}{c}\text { Av } \\
\text { speed }\end{array}$} \\
\hline & $0.5-2.0$ & $\begin{array}{r}\text { For } \\
2.1-3.5\end{array}$ & $\begin{array}{c}\text { winds having } \\
3.6-5.5\end{array}$ & $\begin{array}{c}\text { speeds (in } \\
5.6-9.0\end{array}$ & $\begin{array}{l}\mathrm{m} / \mathrm{s}) \text { of : } \\
9.1-12.0\end{array}$ & $>12$ & $\begin{array}{l}\text { Totals by } \\
\text { direction }\end{array}$ & \\
\hline \multicolumn{9}{|c|}{ Stability Category $A$} \\
\hline $\mathbf{N}$ & 0.40 & 1.41 & 0.98 & 0.15 & 0.0 & 0.0 & 2.94 & 3.40 \\
\hline NNE & 0.27 & 1.23 & 1.97 & 0.78 & 0.02 & 0.0 & 4.27 & 4.37 \\
\hline $\mathrm{NE}$ & 0.51 & 0.98 & 1.23 & 0.38 & 0.0 & 0.0 & 3.10 & 3.79 \\
\hline ENE & 0.42 & 0.42 & 0.51 & 0.18 & 0.0 & 0.0 & 1.53 & 3.49 \\
\hline $\mathbf{E}$ & 0.36 & 0.67 & 0.47 & 0.09 & 0.0 & 0.0 & 1.59 & 3.22 \\
\hline ESE & 0.58 & 0.40 & 0.47 & 0.11 & 0.0 & 0.0 & 1.56 & 3.07 \\
\hline $\mathrm{SE}$ & 0.80 & 0.42 & 0.29 & 0.09 & 0.0 & 0.0 & 1.60 & 2.60 \\
\hline SSE & 0.45 & 0.36 & 0.43 & 0.07 & 0.0 & 0.0 & 1.31 & 3.08 \\
\hline $\mathbf{s}$ & 0.58 & 0.33 & 0.47 & 0.22 & 0.0 & 0.0 & 1.60 & 3.37 \\
\hline SSW & 0.42 & 0.85 & 0.83 & 0.67 & 0.0 & 0.0 & 2.77 & 4.18 \\
\hline SW & 0.31 & 0.74 & 0.38 & 0.25 & 0.0 & 0.0 & 1.68 & 3.58 \\
\hline WSW & 0.15 & 0.15 & 0.04 & 0.0 & 0.0 & 0.0 & 0.34 & 2.32 \\
\hline $\mathbf{W}$ & 0.0 & 0.0 & 0.0 & 0.0 & 0.0 & 0.0 & 0.0 & 0.0 \\
\hline WNW & 0.04 & 0.04 & 0.0 & 0.0 & 0.0 & 0.0 & 0.08 & 2.03 \\
\hline $\mathrm{NW}$ & 0.13 & 0.15 & 0.13 & 0.0 & 0.0 & 0.0 & 0.41 & 2.86 \\
\hline NNW & 0.33 & 0.80 & 0.18 & 0.04 & 0.0 & 0.0 & 1.35 & 2.79 \\
\hline Total & 5.75 & 8.95 & 8.38 & 3.05 & 0.02 & 0.0 & 26.13 & 3.55 \\
\hline
\end{tabular}




\begin{tabular}{|c|c|c|c|c|c|c|c|c|}
\hline$\left\langle 7^{\circ} \eta\right.$ & $\ddot{z} L \cdot z$ & 70.0 & 20.0 & Or. 0 & $\tau \varepsilon * T$ & $18^{\circ} 0$ & $\mathrm{CT}^{\circ} \mathrm{O}$ & $a N$ \\
\hline $2 L \cdot S$ & It $c$ & $60^{\circ} 0$ & $\mathrm{II} \cdot 0$ & $25^{\circ} \mathrm{Z}$ & $9 S^{\circ} \mathrm{I}$ & $86^{\circ} 0$ & $S I^{\circ} 0$ & JNN \\
\hline $8 E^{\cdot} 9$ & $80^{\circ} \mathrm{S}$ & $t \tau^{\circ} 0$ & $8 \tau^{\circ} 0$ & $06^{\circ} z$ & $z \cdot E^{*} \mathrm{I}$ & $\angle \nabla^{\circ} 0$ & 50.0 & $\mathbf{N}$ \\
\hline \multicolumn{9}{|c|}{ 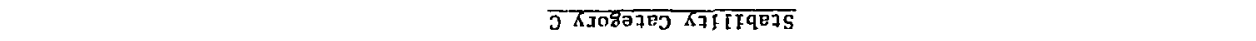 } \\
\hline$I Z \cdot \eta$ & $\varsigma 乙 \cdot 8 T$ & 0.0 & $20^{\circ} 0$ & $6 \varepsilon \cdot 7$ & $80 \cdot 9$ & $\varepsilon 9^{\circ}$ & $\varepsilon I^{\cdot} E$ & โE70 L \\
\hline$O T^{\circ} \varepsilon$ & $\varsigma E^{*} \mathrm{~L}$ & 0.0 & 0.0 & $50^{\circ} 0$ & $9 \varepsilon \cdot 0$ & $\varsigma 9^{\circ} 0$ & $62 \cdot 0$ & MNN \\
\hline$s 9^{\circ} z$ & $\mathbf{T S}{ }^{\circ} 0$ & 0.0 & 0.0 & 0.0 & $\angle 0^{\circ} 0$ & IE:0 & $\varepsilon I \cdot 0$ & $M N$ \\
\hline 0.0 & 0.0 & 0.0 & 0.0 & 0.0 & 0.0 & 0.0 & 0.0 & MNM \\
\hline 0.0 & 0.0 & $0 \cdot 0$ & 0.0 & 0.0 & 0.0 & 0.0 & 0.0 & $M$ \\
\hline $90^{\circ} \hat{c}$ & $\varsigma I^{\cdot} 0$ & 0.0 & 0.0 & 0.0 & 70.0 & $60^{\circ} 0$ & $20^{\circ} 0$ & MSM \\
\hline$\mapsto \varepsilon^{*} \varepsilon$ & $\mathbf{I} \boldsymbol{\top} \cdot \mathrm{T}$ & 0.0 & $0 \% 0$ & $50^{\circ} 0$ & $\varepsilon \varsigma \cdot 0$ & {$[L \cdot 0$} & $22 \cdot 0$ & MS \\
\hline$โ \sigma^{\circ} \varepsilon$ & $0 \sqcap \cdot 2$ & 0.0 & 0.0 & $\varepsilon \varsigma^{\circ} 0$ & $\varepsilon 9^{\circ} 0$ & $I \angle L^{\circ} 0$ & $\varepsilon S^{\circ} 0$ & MSS \\
\hline$z L^{*} \varepsilon$ & $\varepsilon Z \cdot \tau$ & 0.0 & $0^{\circ} 0$ & $\angle Z \cdot O$ & $6 Z^{\circ} 0$ & $62^{\circ} 0$ & $8 \varepsilon * 0$ & $\mathbf{s}$ \\
\hline$\varepsilon \tau^{*} \varepsilon$ & $I E^{\prime} 0$ & 0.0 & 0.0 & $70^{\circ} 0$ & $\angle 0^{\circ} 0$ & $\angle 0^{\circ} 0$ & $\varepsilon T \cdot 0$ & ass \\
\hline$Z L^{\circ}[$ & $6 \varepsilon^{-} 0$ & $0^{\circ} 0$ & 0.0 & $20 \% 0$ & 0.0 & $70 \cdot 0$ & $\varepsilon \varepsilon^{*} 0$ & AS \\
\hline $96^{\circ} z$ & $z \mapsto \cdot 0$ & 0.0 & 0.0 & $\angle 0^{\circ} 0$ & $\angle 0^{\circ} 0$ & $70^{\circ} 0$ & $\eta Z^{-} 0$ & ISA \\
\hline $56^{\circ} z$ & $2 \varepsilon^{\circ} 0$ & 0.0 & 0.0 & $\varsigma 0^{\circ} 0$ & $70^{\circ} 0$ & $\angle 0^{\circ} 0$ & $9 \mathrm{I}^{\circ} 0$ & 3 \\
\hline $8 z^{\prime} \varepsilon$ & 59.0 & 0.0 & 0.0 & $60^{\circ} 0$ & $60 \%$ & IE $=0$ & $9 T^{\circ} 0$ & INA \\
\hline $06^{\circ}$ & $89^{\circ} \mathrm{I}$ & 0.0 & 0.0 & $9 S^{*} 0$ & $\angle 9^{\circ} 0$ & $\eta E^{*} 0$ & IT ${ }^{\circ} 0$ & $\mathrm{gN}$ \\
\hline $9 I^{\circ} \mathrm{s}$ & $\varepsilon 9 \cdot 7$ & 0.0 & 0.0 & $z L^{\circ} I$ & $90 \cdot 2$ & $85^{\circ} 0$ & $\angle \bar{C}^{\prime} 0$ & INN \\
\hline 80.5 & $0 L^{\cdot} Z$ & 0.0 & 20.0 & $76^{\circ} 0$ & $91 \cdot \mathrm{t}$ & こヴ0 & $9 \Gamma^{\circ} 0$ & $\mathbf{N}$ \\
\hline
\end{tabular}

-panutguos " 6 a [quJ. 
Table 9, cont inued.

\begin{tabular}{|c|c|c|c|c|c|c|c|c|}
\hline ENE & 0.45 & $1.5 \mathrm{~b}$ & 0.85 & 0.18 & 0.0 & 0.0 & 3.04 & 3.33 \\
\hline E & 0.24 & 0.38 & 0.27 & 0.09 & 0.0 & 0.0 & 0.98 & 3.32 \\
\hline ESE & 0.29 & 0.25 & 0.20 & 0.02 & 0.0 & 0.0 & 0.76 & 2.79 \\
\hline SE & 0.27 & 0.02 & 0.07 & 0.07 & 0.0 & 0.0 & 0.43 & 2.84 \\
\hline SSE & 0.51 & 0.09 & 0.15 & 0.07 & 0.0 & 0.0 & 0.82 & 2.54 \\
\hline $\mathbf{s}$ & 0.20 & 0.36 & 0.98 & 0.51 & 0.07 & 0.11 & 2.14 & 4.82 \\
\hline SSW & 0.18 & 0.63 & 0.62 & 0.56 & 0.05 & 0.0 & 2.04 & 4.62 \\
\hline su & 0.47 & 0.60 & 0.36 & 0.04 & 0.0 & 0.0 & 1.47 & 2.85 \\
\hline WSH & 0.11 & 0.20 & 0.02 & 0.0 & 0.0 & 0.0 & 0.33 & 2.39 \\
\hline w & 0.0 & 0.0 & 0.0 & 0.0 & 0.0 & 0.0 & 0.0 & 0.0 \\
\hline HNW & 0.04 & 0.07 & 0.0 & 0.0 & 0.0 & 0.0 & 0.11 & 2.24 \\
\hline NW & 0.16 & 0.25 & 0.07 & 0.0 & 0.0 & 0.0 & 0.48 & 2.34 \\
\hline NNW & 0.02 & 0.22 & 0.18 & 0.09 & 0.02 & 0.0 & 0.53 & 4.39 \\
\hline Total & 3.27 & 6.89 & 7.97 & 7.47 & 0.45 & $0.2 \%$ & 26.29 & 4.66 \\
\hline \multicolumn{9}{|c|}{ 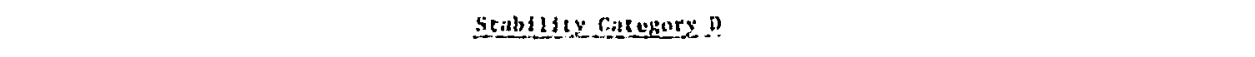 } \\
\hline $\mathrm{s}$ & 0.0 & 0.15 & 0.42 & 0.81 & 0.18 & 0.04 & 1.60 & 6.71 \\
\hline XYE & 0.13 & 0.67 & 0.91 & 0.62 & 0.04 & 0.16 & 2.53 & 3.35 \\
\hline NE & 0.36 & 3.57 & 1.35 & 0.45 & 0.05 & 0.02 & 3.79 & 3.97 \\
\hline ENE & 0.89 & 4,00 & 1.95 & 0.20 & 0.0 & 0.02 & 7.06 & 3.25 \\
\hline$F$ & 0.73 & 1.63 & 0.33 & 0.07 & 0.0 & 0.0 & 2.51 & 2.69 \\
\hline ESP: & 0.20 & D. 40 & 0.22 & 0.16 & 0.02 & 0.0 & 1.00 & 3.75 \\
\hline st: & 0.09 & 0.07 & 0.13 & 0.02 & 0.0 & 0.0 & 0.31 & 3.37 \\
\hline Ssi: & 0.42 & 0.34 & 0,43 & 0.04 & 0.0 & 0.0 & 1.23 & 3.03 \\
\hline
\end{tabular}




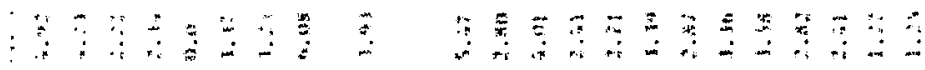

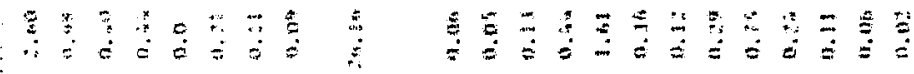

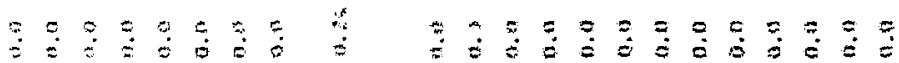

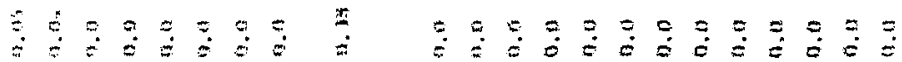

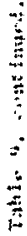

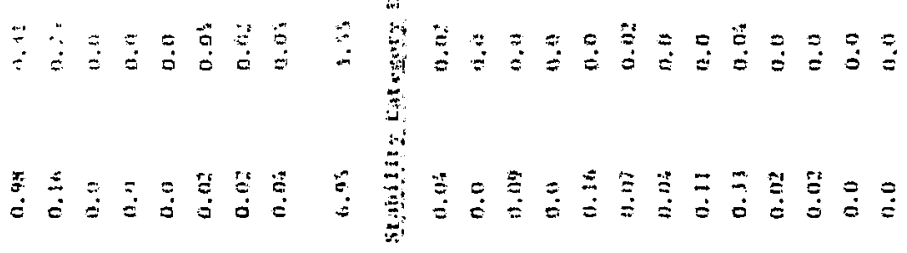

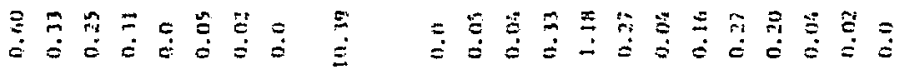

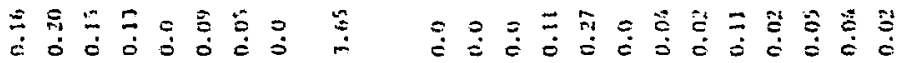

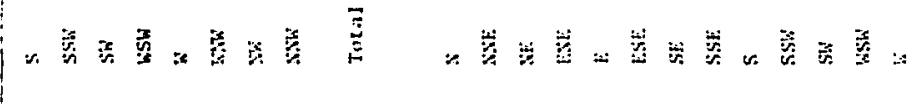




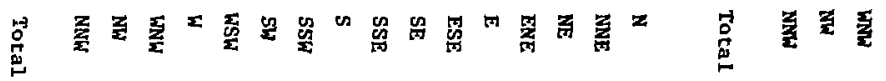

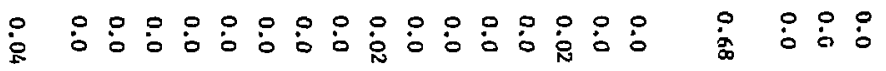

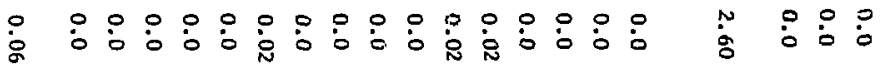

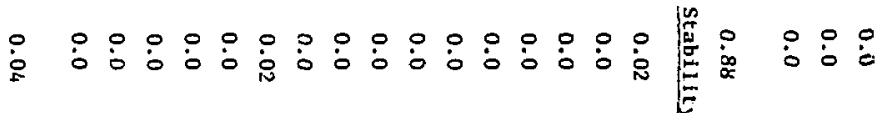

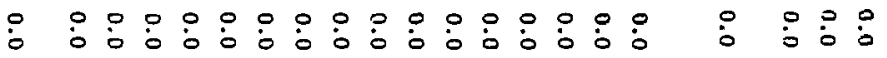

$\begin{array}{lllllllllllllllllllll}0 & 0 & 0 & 0 & 0 & 0 & 0 & 0 & 0 & 0 & 0 & 0 & 0 & 0 & 0 & 0 & 0 & 0 & 0 & 0 & 0 \\ 0 & 0 & 0 & 0 & 0 & 0 & 0 & 0 & 0 & 0 & 0 & 0 & 0 & 0 & 0 & 0 & 0 & 0 & 0 & 0\end{array}$

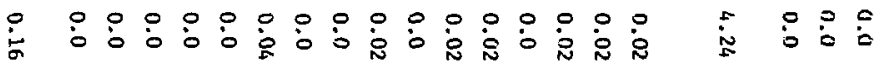

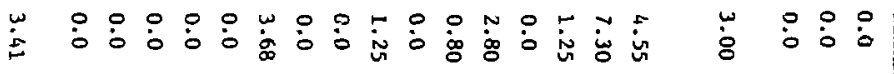


in Table 6. Tables 7, 8, and 9 show the relialive uniformity of average wind speed between stability :itegorles.

\section{Also, for certain wind ditec-} tions, bigher wind-speed ranges cecur relatively rrequent $y$ in stahility catchories that are tradtionally thought to occur at low windspeceds. In part, Lhis is dus to the mechanics of extracting stabilletes from windspeced tratsis. Tlie prosent state of the art preludes using more sophlsticisted techniques. A sumeary of these stibility wind-rose tables for winter and summer is shown in Tible 10.
HLMIDITY ANB FoG

The only humidfly records laken routingly at ats arc made at iuccat Flate. Fine climatologlcal stumary, Tilb] I, shows [yplcally high humfat ies in early morning, with significanty lower readyugs in the afternoon. The reported averages reflect the descrt climate - in annual-average $4: 00$ a.m. value of $53 \%$ and a $4: 00 \mathrm{pem}$. value of $23 \%$. Fog is a rare occurrence at NTS, Fog is present feser than six days per yent in smithern Nevada. 5 Most of these cases occur in winter, but the crind is not pronounced.

\section{Program of Onsite Meteorological Messurements}

\section{$\Lambda$ C.LINET 013-1 wind-sensing} system is operated on a 10-m tower at "Area 410 Basin Nh'." This location is northwest of the LLL assembly buildings. The starting speed of the CLMMET system is less than 0.5 $\mathrm{m} / \mathrm{s}$. Recordings are made on strip charts and the mint is serviced every two weeks by National oceanic and Atmospherle Admintstration personnel.

\section{Long-Term (Routine) Diffusion Estinates}

Due to the nature of Area 410 activitles, there are no routine relsages of fontzing radiation or other potentially harmful pollutants. Hence, no estimates of long-term diffuston were made in our study. 


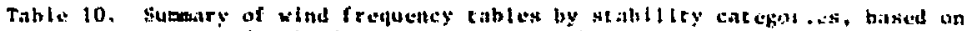

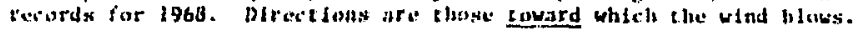

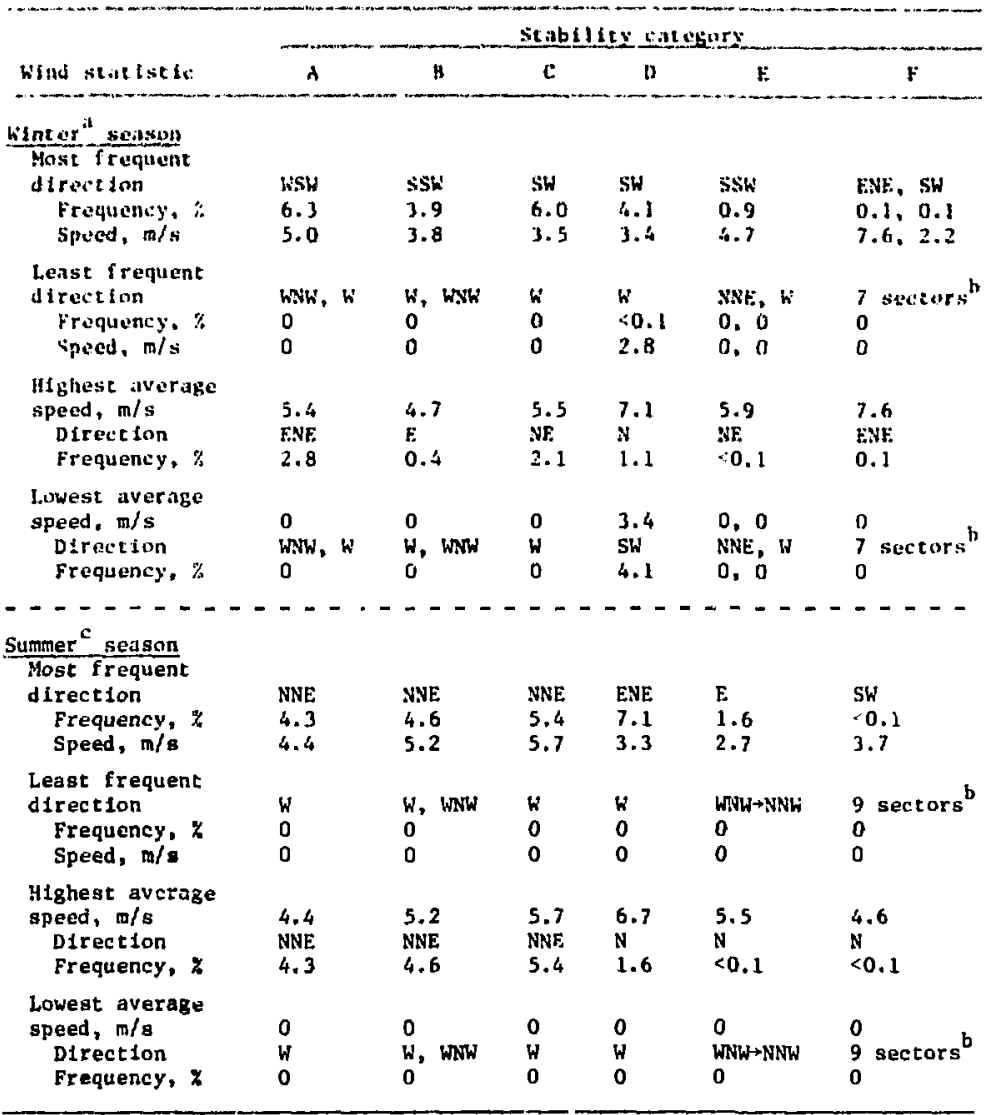

${ }^{a}$ October through February.

birection divided into 16 sector each $22.5^{\circ}$ wide.

March through September. 


\section{Short-Term (Accident) Diffusion Estimates}

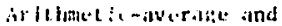

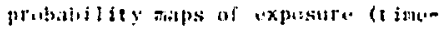

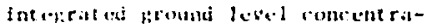

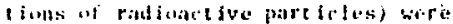

abtolined from the fustantaneous point sosurae (15's) rolle develuped by

Pelersin. the input walla nere the cower whads absorved neat super kuklat. The benetialized diffusion equation

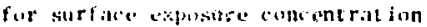
ancer an explosion's rloted conter line is

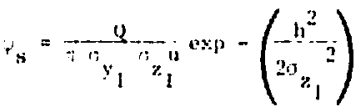

wherc is = stiriace exposiure, in unlts of 1$) \cdot \mathrm{s} / \mathrm{m}^{3}$.

$$
\begin{aligned}
Q= & \text { sourec term, assumed } \\
& \text { unity in this report. }
\end{aligned}
$$

$\sigma_{y_{1}}=$ Instantaneous crosswind deviation, in $\mathrm{m}$.

$\begin{aligned} \sigma_{z_{I}}= & \text { Instantanecas vertical } \\ & \text { standard deviation, in } m .\end{aligned}$

u = hor 1zontal windspeed at cloud center, in $\mathrm{m} / \mathrm{s}$.

h - height of cloud center above ground, in $\mathrm{m}$.
The lps rode uste walton's scale-tidundent difiasion approach $i$ determine $s_{i} ; \nabla_{i}$ is obtatined irot che equat fon $\sigma_{2 !}=\left(2 k_{x} t\right)^{1 / 2}$, where $k_{z}$, che vereical diffusion cuefflciont, is d stsbility-dependent inpus parameter, and $t$ is travel t ime doribind. Sald in from ubscrutions of explosions were used to decermine the helglit and geomety of the stabfllaed cloud. The valculations in this secefon bul lade surface exposures and depositfon trom a "puff" of gases and particles that have no apprectable lali velocity (radius less than 05 im). Fallout of larger particles, which could produce substantially higher depositions, is not included.

Three types of accidents have been postulated, and this report presents contuur maps of radioactive exposure presented for all types. These aceldents are:

- The dispersal of plutonium by detonation of $68 \mathrm{~kg}$ (150 1b) of high explosives (HE).

- a criticality accilent Involving $10^{19}$ issions of uranfum-235 (equivilent to about a 140-1b high-explosives detonation), and

- an inadvertent 100-ton fission explosion. 
HE AND CRITICALITY ACCIDENTS

For accident \& (a) and (b), the explostve ylejds are nearly equal. Hence, It is astiumed that their rosulting cloud geometries are the same, and one set of exposure-contour
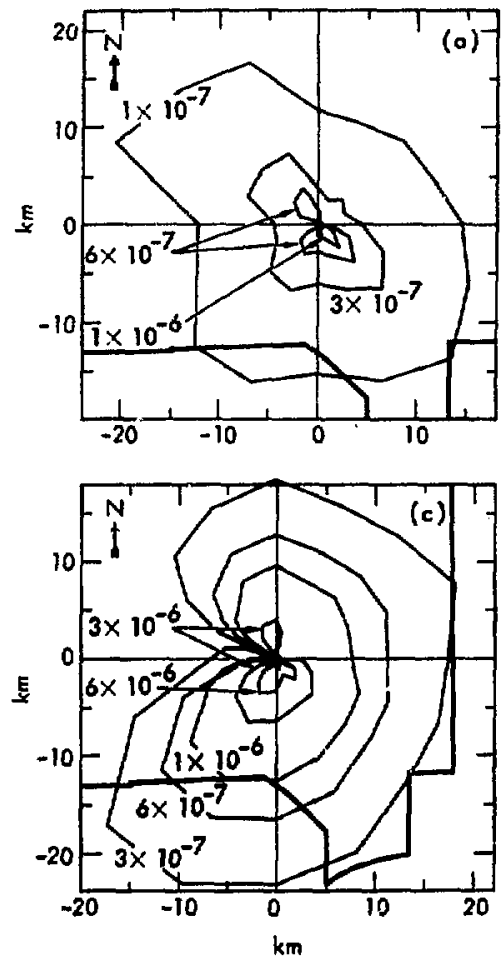

mapg covering both these aceldents is presented ft Fisti, 6, 7, and 8 , fiach

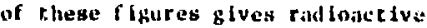
exposures calculation on the basts of annual, wines, and summat wind daxa. respectively. Half wi the maps on each tine period show the artelmet is
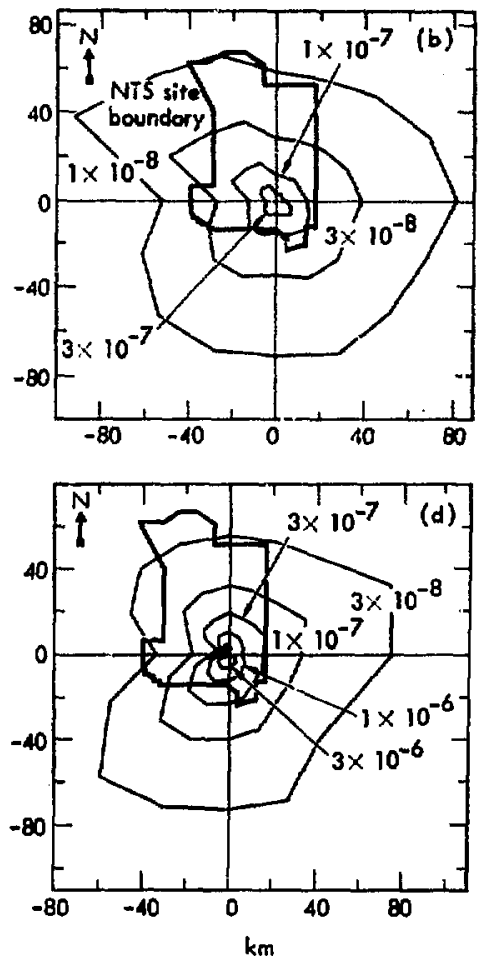

Fig. 6. Relative exposures (In $s / m^{3}$ ) around Area 410 for HE or critcility accident, baed on annual sind data: (a) and (b) average exposures at near and far perapectiven, respectively; (c) and (d) exposure 11mits exceeded $5 x$ of the time at near and far perspectives. respect Ively. 

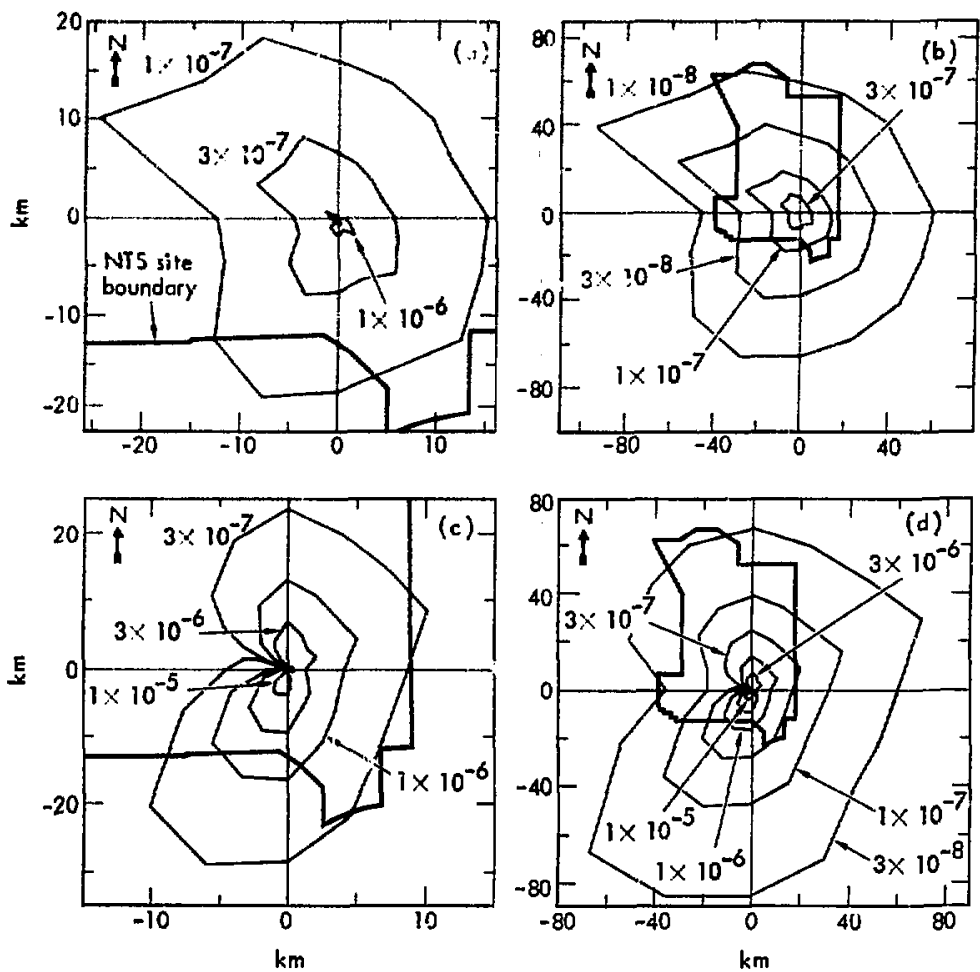

Fis. 7. Relative exposures (In $\mathrm{s} / \mathrm{m}^{3}$ ) around Area 410 for HE or erfticaljty accidert, based on winter (October-February) wind data: (a) and (b) average exposures at near and far perspectives, respectively; (c) and (d) exposure $11 \mathrm{mits}$ exceeded $5 \%$ of the $t$ ime at near and far perspectiven, respectively. 

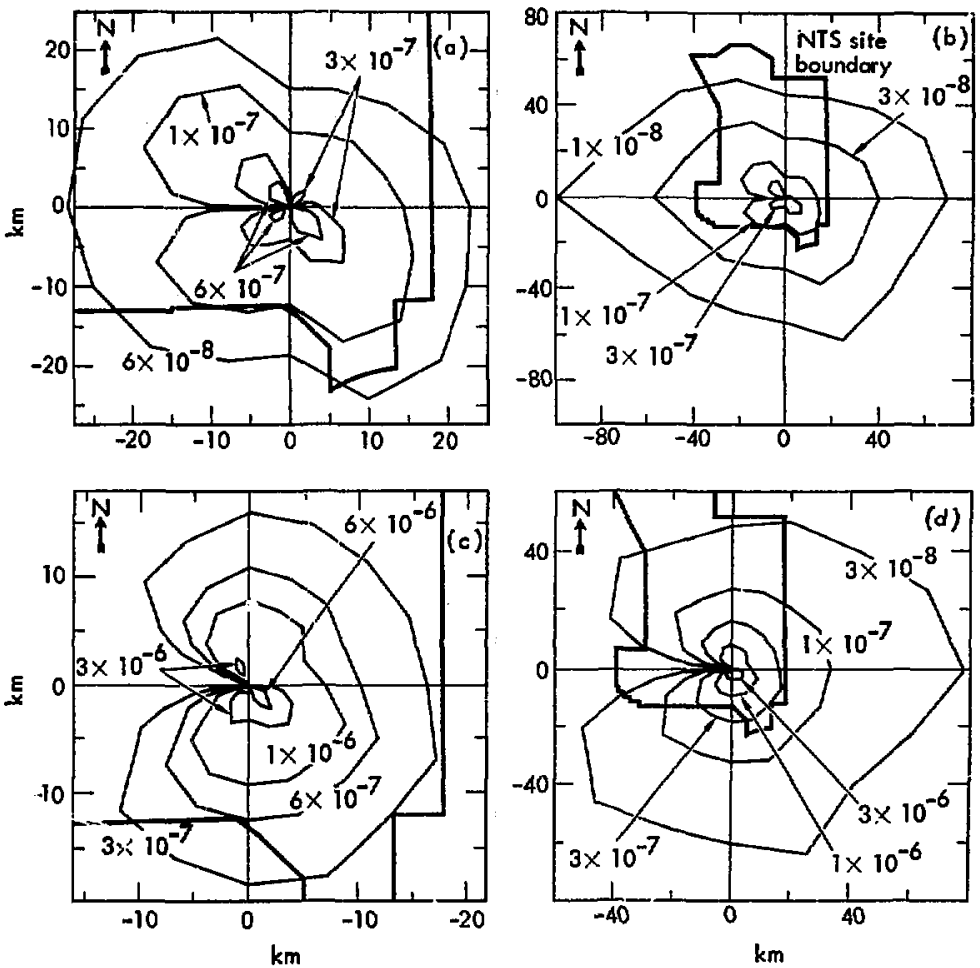

FIg. 8. Relative exposures (In $\mathrm{s} / \mathrm{m}^{3}$ ) around Area 410 for HE or criticality accident, based on summer (March-September) wind data: (a) and (b) average exposures at near and far perspectives, respectively; (c) and (d) exposure 11mita exceeded $5 \%$ of the $t$ ime at near and far perspectives, respectively. 
average exposures, and the other half were computed allowing a $5 \%$ probability that the specified exposures will bo exceded. The liater maps are prepared by assuming a lognormal distribution of exposure and omitting the top $5 \%$ of the distribution. Hence, one random accident out of twenty will result in exposures greater than shown on the map. Both close-up and distant maps are presented for each of the average and $5 \%$ cases within each time period, with the distant map shuwing exposures as far as $83 \mathrm{~km}$ irom Area 410 . Also, the NTS :atte boundary is sketched on the contour maps.

The IPS code depletes the puff with distance according to the deposition velocity, which was assumed to be $0.01 \mathrm{~m} / \mathrm{s}$. The product of the exposure and the deposition velority yields deposition, in units of $\mathrm{m}^{-2}$. Hence, multiplying the exposure-contour values by $0.01 \mathrm{~m} / \mathrm{s}$ glves surface-deposition contour values. A1l atmospheric stability categorles, weighted by their relative frequencies, were Inclind ed by the coda when it calculated the maps. The specific exposure values for each contour are elliered on the diagrams.

The annual average maps have a nearly clrcular distribution, with a slight dimunition of exposures towand the west and west-southwest. The annual 5\% maps show a northeast- southwest elongation with diminished values toward the west-northwest. The average and $5 \%$ exposure contours of winter are close to those of the annual mass ¿a both shape and magnitude. The northeast-southvest elongaticn is more pronounced in the winter $5 \%$ maps. This is due to wind and stablifty conditions that favor higher exposures when the wind direction is from the $\mathrm{NE}$ and $\mathrm{SW}$.

In the sumer, the average exposure pattern shows a general east-west eiongation. However, the $5 \%$ contours for the same period are nearly :ircular except for zero exposure values toward the west. The zero values are due to the fact that only one wind toward the west (from the east) was observed in the summer of 1908 . The probability routine in the IPS code is unable tc treat one case.

The direction toward $w^{i} l i c h$ exposure at the site buundary is greatest is raferred to as the "critical azimuth." Table 11 presents the directions and relative exposures where the critical azimuth crosses the site boundary, as well as those at the closest site boundary (to the soutis). Note that the critical-azimuth values are less than $20 \%$ greater thin exposures to the south.: Exposure values at various distances to $100 \mathrm{~km}$ are presented in Table 12. 
Table 11. Relative exposures at the site boundary's intersection with the critical azimuth and at the closest point of tire boundary that would result from an $H E$ or criticality explosion.

\begin{tabular}{|c|c|c|c|c|c|c|}
\hline & \multicolumn{3}{|c|}{ Arithmetic av } & \multicolumn{3}{|c|}{$95 \%$-probable 1 imit } \\
\hline & Annual & Winter & Summer & Annual & Winter & Summer \\
\hline $\begin{array}{l}\text { Direction of the } \\
\text { critical azimuth }\end{array}$ & SSW & SSW & $\mathrm{SE}$ & ssw & SSW & $\mathbf{S}$ \\
\hline Exposure, $s / \pi^{3}$ & $1.5 \times 10^{-7}$ & $1.8 \times 10^{-7}$ & $1.0 \times 10^{-7}$ & $1.1 \times 10^{-6}$ & $1.7 \times 10^{-6}$ & $5.7 \times 10^{-7}$ \\
\hline $\begin{array}{l}\text { Direction of the } \\
\text { closest site } \\
\text { boundary }\end{array}$ & $\mathbf{S}$ & $\mathbf{S}$ & $\mathbf{S}$ & $\mathrm{S}$ & $\mathbf{S}$ & $\mathrm{s}$ \\
\hline Exposure, $s / \mathrm{m}^{3}$ & $1.3 \times 10^{-7}$ & $1.6 \times 10^{-7}$ & $9.4 \times 10^{-8}$ & $9.5 \times 10^{-7}$ & $1.5 \times 10^{-6}$ & $5.7 \times 10^{-7}$ \\
\hline
\end{tabular}

Deposition values (in units of $\mathrm{m}^{-2}$ ) are $1 / 100$ of exposure values.

100-TON FISSION EXPLOSION

A 100-ton fission explosion would generate a stabilized cloud center at about $1800 \mathrm{~m}$ above the surface. This altitude is well bevond the valid range for the power-1aw wind profile, used to adjust wind speeds at $10 \mathrm{~m}$ above the surface to those at the approx 200-m cloud-center height for an HE or criticality accident. Hence, we prepared new input data, using the 1968 upper-air wind conditions observed twlce-daily at Yucca Fjat. These winds are representative of those at Area 410. The corresponding stability categories were determined from these obsezvations and the near-surface to cloud, centertemperature profile.
The frequencies and average speeds of these upper-air winds as a Function of the direction toward which the wind blows are presented in Table 13. The average speeds are generally greater than those of $10-m$ winds (listed in Table 4), but by less than a fastor of two. Winter wind speeds are greater than summer wind speeds. Such behavior is cansistent with usual winds in middle latitudes. The wind frequencies at $1800 \mathrm{~m}$ also siffer from those at $10 \mathrm{~m}$. For the $1800-\pi$ winds, the most frequent directions are toward the SSW and SW in both winter and summer. (The 10-m winds show a pronounced roversal toward the NNE in summer.)

The Erequencies of stability categorles affecting a 100-ton fission accident are shown in Table 
Table 12. Relative cxposures $\left(s / \mathrm{m}^{3}\right)$ along the critical azimurh at stee boundary and other points at distances to $1.00 \mathrm{~km}$; for HE or critfeality accidert.

\begin{tabular}{|c|c|c|c|c|c|c|c|c|c|}
\hline \multirow[b]{2}{*}{ Type of exposure } & \multirow[b]{2}{*}{$\begin{array}{c}\text { Critical } \\
\text { azimuth }\end{array}$} & \multicolumn{8}{|c|}{ Distance along critical azimuth $-\mathrm{km}$} \\
\hline & & 1 & 2 & at & $\begin{array}{c}\text { distances } \\
10\end{array}$ & (in $\underset{\mathrm{si}^{\mathrm{a}}}{\mathrm{a}}$ of & 20 & 50 & 100 \\
\hline \multicolumn{10}{|l|}{ Based on annuitl data } \\
\hline arithnetic average & SSW & $7.8 \times 10^{-7}$ & $7.5 \times 10^{-7}$ & $4.3 \times 10^{-7}$ & $2.1 \times 10^{-7}$ & $1.5 \times 10^{-7}$ & 8. $.3 \times 10^{-8}$ & $2.0 \times 10^{-8}$ & $5.8 \times 10^{-9}$ \\
\hline 95\%-probable limit & SSiw & $7.2 \times 10^{-6}$ & $8.4 \times 10^{-6}$ & $4.7 \times 10^{-6}$ & $1.7 \times 10^{-6}$ & $1.1 \times 10^{-6}$ & $4.5 \times 10^{-7}$ & $7.5 \times 10^{-3}$ & $1.8 \times 10^{-8}$ \\
\hline \multicolumn{10}{|l|}{ Based on winter data } \\
\hline arithetic average & SSW & $1.0 \times 10^{-6}$ & $9.3 \times 10^{-7}$ & $5.3 \times 10^{-7}$ & $2.5 \times 10^{-7}$ & $1.8 \times 10^{-7}$ & $1.0 \times 10^{-7}$ & $2.3 \times 10^{-8}$ & $6.6 \times 10^{-9}$ \\
\hline $95 \%$-probable limic & $5 S w$ & $1.2 \times 10^{-5}$ & $1.4 \times 10^{-5}$ & $8.3 \times 10^{-6}$ & $2.9 \times 10^{-6}$ & $1.7 \times 10^{-6}$ & $7.5 \times 10^{-7}$ & $1.0 \times 10^{-7}$ & $2.5 \times 10^{-8}$ \\
\hline \multicolumn{10}{|l|}{ Based on summer data } \\
\hline arithmetic average & $S E$ & $2.2 \times 10^{-6}$ & $1.6 \times 10^{-6}$ & $7.7 \times 10^{-7}$ & $2.9 \times 10^{-7}$ & $1.0 \times 10^{-7}$ & $9.0 \times 10^{-8}$ & $2.1 \times 10^{-8}$ & $5.8 \times 10^{-9}$ \\
\hline 95\%-probable 1imit & $s$ & $3.3 \times 10^{-6}$ & $3.6 \times 10^{-6}$ & $2.2 \times 10^{-6}$ & $8.6 \times 10^{-7}$ & $5.7 \times 10^{-7}$ & $2.5 \times 10^{-7}$ & $4.5 \times 10^{-8}$ & $1.2 \times 10^{-8}$ \\
\hline
\end{tabular}

${ }^{a} \mathrm{SB}=$ Critical a\%imuth site boundary: $13.5 \mathrm{~km}$ to $\mathrm{SSW}, 12.5 \mathrm{~km}$ to $\mathrm{s}, 19 \mathrm{~km}$ to $\mathrm{SE}$. 
Table 13. Frequency and avarage speeds vs wind direction of winds at $1800 \mathrm{~m}$ above ground affecting a 100-ton fission explosion at Area 410 (based on 1968 data).

\begin{tabular}{|c|c|c|c|c|c|c|}
\hline \multirow[b]{2}{*}{$\begin{array}{l}\text { Direction } \\
\text { toward which } \\
\text { wind blows }\end{array}$} & \multicolumn{2}{|c|}{ Annual } & \multicolumn{2}{|c|}{ Winter } & \multicolumn{2}{|c|}{ Summer } \\
\hline & $\begin{array}{c}\text { Frequency, } \\
\% \%\end{array}$ & $\begin{array}{l}\text { Av } \\
\text { speed, } \\
\mathrm{m} / \mathrm{s}\end{array}$ & $\underset{\%}{\text { Erequency }}$ & $\begin{array}{l}\text { Av } \\
\text { speed, } \\
\mathrm{m} / \mathrm{s}\end{array}$ & $\underset{\%}{\text { Frequency, }}$ & $\begin{array}{c}\text { Av } \\
\text { speed } \\
\mathrm{m} / \mathrm{s}\end{array}$ \\
\hline $\mathrm{N}$ & 6.0 & 8.2 & 6.5 & 9.4 & 5.7 & 7.4 \\
\hline NNE & 4.6 & 8.7 & 5.4 & 9.1 & 4.0 & 8.4 \\
\hline $\mathrm{NE}$ & 4.1 & 7.8 & 6.2 & 8.8 & 2.9 & 6.4 \\
\hline ENE & 1.8 & 6.0 & 1.5 & 9.0 & 1.9 & 4.5 \\
\hline $\mathrm{E}$ & 1.3 & 5.6 & 1.9 & 6.8 & 1.0 & 4.0 \\
\hline ESE & 0.7 & 5.4 & 0.8 & 3.0 & 0.7 & 7.0 \\
\hline$S E$ & 1.5 & 4.4 & 0.8 & 3.5 & 1.9 & 4.6 \\
\hline SSE & $4 \cdot 3$ & 5.7 & 2.7 & 8.3 & 5.2 & 4.8 \\
\hline $\mathbf{S}$ & 6.6 & 6.7 & 2.7 & 3.7 & 9.0 & 7.2 \\
\hline SSW & 16.9 & 9.3 & 10.8 & 12.2 & 20,7 & 8.4 \\
\hline SW & 15.9 & 9.6 & 15.8 & 13.2 & 16.0 & 7.4 \\
\hline WSH & 7.8 & 6.6 & 8.5 & 8.0 & 7.4 & 5.6 \\
\hline$w$ & 7.1 & 6.4 & 5.8 & 8.3 & 7.9 & 5.6 \\
\hline WNW & 5.6 & 6.5 & 6.9 & 7.8 & 4.8 & 5.2 \\
\hline $\mathrm{NW}$ & 10.3 & 8.4 & 14.6 & 9.8 & 7.6 & 6.8 \\
\hline NNW & 5.6 & 9.2 & 9.2 & 10.8 & 3.3 & 6.4 \\
\hline$\frac{\text { All }}{\text { directions }}$ & 100. & 7.2 & 100. & 8.2 & 100 & 6.2 \\
\hline
\end{tabular}

14. On an annual basis, "D" (neutral) stablilty occurs over $60 \%$ of the time at $1800 \mathrm{~m}$ above ground. In the winter, essentially all the stabllities are "D" or " $E$ ", with "E" occurring almost $60 \%$ of the time, wh1le in the summer " $D$ " stability predominates over $70 \%$ of the time. More stable conditions are to be expected in winter, particularly in a desert environmenr.

A comparison of Table 14 with

Table 6 (based on 10 $-\mathrm{m}$ winds) indicates considerably higher Erequencles of stable conditions affectir:g the 100-ton nuclear accident. This is consistent with daytime abservations of lapse rate 
Table 14. lirequency of stability categories for 100-ton fisston explosion at Area 410 (based on winds at $1800 \mathrm{~m}$ above ground).

\begin{tabular}{cccc}
\hline $\begin{array}{l}\text { Stablity } \\
\text { Category }\end{array}$ & Frequency, $\%$ & $\begin{array}{c}\text { Winter } \\
\text { (Oct-Feb) }\end{array}$ & $\begin{array}{c}\text { Summer } \\
\text { (Niar-Sept) }\end{array}$ \\
\hline A & 0.4 & 0 & 0.7 \\
$B$ & 4.0 & 0 & 6.4 \\
C & 6.9 & 0 & 11.2 \\
D & 61.3 & 41.9 & 73.3 \\
E & 27.2 & 57.3 & 8.3 \\
F & 0.2 & 0.4 & 0 \\
\hline
\end{tabular}

vs height. Super-adiabatic lapse races (very unstable) often occur near the surface, espectally in a desert environment, At higher levels, the lapse rate usually becomes neutral or slightly stable. Also, it has been observed, upon occasion, that the $\Delta \theta$ method of assessing stabilities (from wind-direction strip charts) has tended to yield stabllities that are too unstable, by about one pasquill category.

The upper-atr winds ware used to calculate relative exposures for anmul, winter, and summer periods for both the arfthmetic average exposure and the $5 \%$ probability that specifted exposures would be exceeded.

Dise to the geometry of the 100ton fisston exploston's stahtlized cloud (at about a helght of $1 E 0 \mathrm{~m}$ above the ground), the gradtent of exposure values along a radial is small for both the arithmetic average and the $5 \%$ calculations. This small gradient is due to the fact that as the cloud moves downind, the surface concentrations of a pollutant decrease while the cloud's time in passing increases at a nearly compensating rate. As a result, the exposure at any point during cloud passage is nearly constant.

Instead of calculating exposure values at intervals so small that they greatly exceed the estimated error of the calculations, 1 is preferable to present the average of exposures out to $100 \mathrm{~km}$, with an Indication of the varfation about the average. This latter approach has been used in Figs, 9-11. The top number within each sector represents the average of exposure values out to $109 \mathrm{~km}$, in units of $10^{-10} \mathrm{~s} / \mathrm{m}^{3}$. 

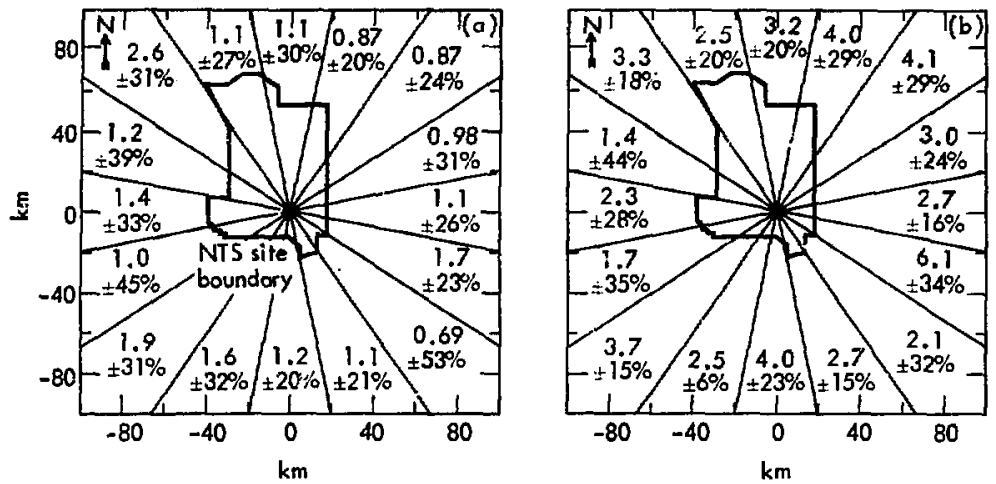

Fig. 9. Relative Exposures ( $1 \mathrm{n} 10^{-10} \mathrm{~s} / \mathrm{m}^{3}$ ) for $100-t o n$ fission accident, based on annual wind data. Percentages indicate variation of exposures within each zone up to $100 \mathrm{~km}$ from Area 410 . Fig. 6a gives the arithmetic average exposures, F1g. 6b gives a $11 \mathrm{mit}$ of exposure exceeded $5 \%$ of the time.
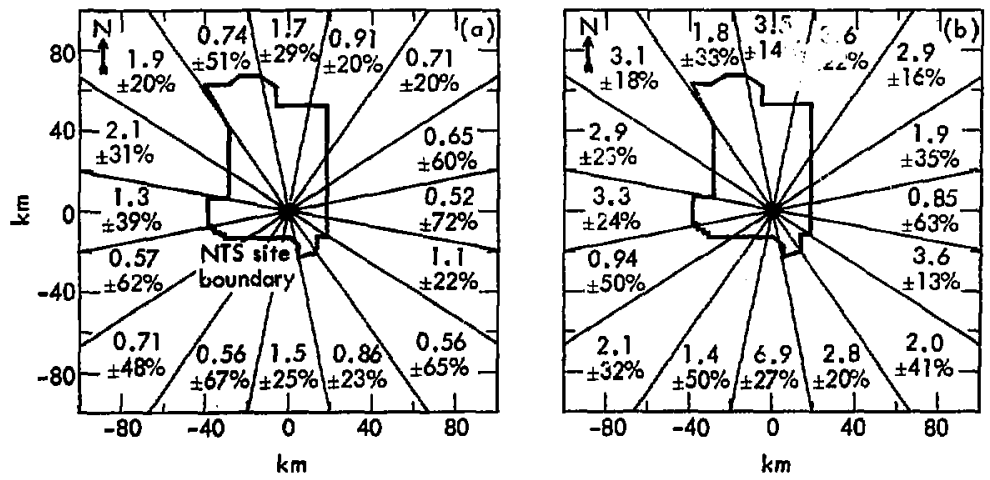

F1 . 10. Relative exposures (In $10^{-10} \mathrm{~s} / \mathrm{m}^{3}$ ) for 100 -ton fission accident, based on winter (October-February) wind data. Percentages indicate variation of exposures within each zone up to $100 \mathrm{~km}$ for Area 410. Fig. 6a glves the arithmetic average exposures; Fig. 6b gives a $11 \mathrm{mit}$ of exposure exceeded $5 \%$ of the time. 

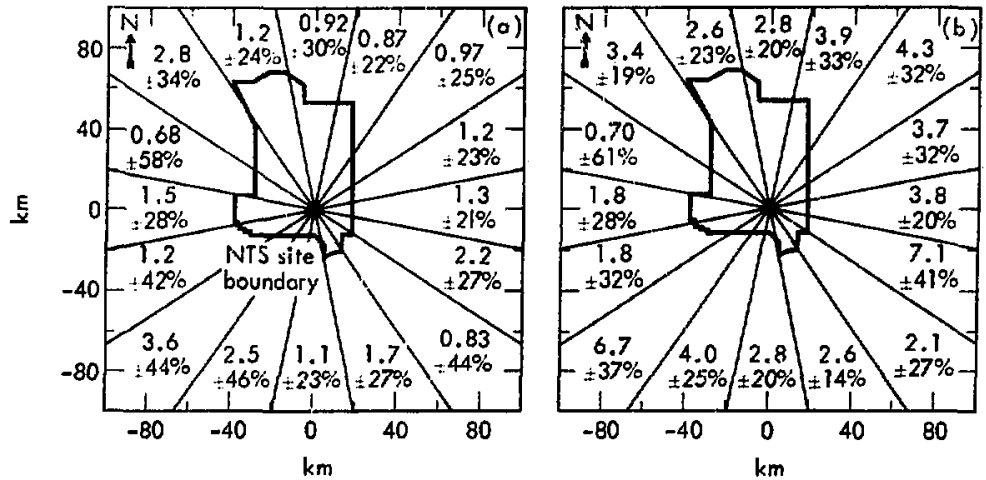

Fif. 11. Relative exposures (in $10^{-10} \mathrm{~s} / \mathrm{m}^{3}$ ) for 100-ton fisston accident, based on sumer (March-September) wind data. Percentages ind lcate virjation of exposures whthin each zone up to $100 \mathrm{kn}$ from Area 410 . Fip. ba gives the artlometic average exposures, $\mathrm{Fig}$. 6b glves a Ifmit nl exposiure exceeded $5 \%$ of the $t$ ine.

The botomn number is the absolute range of calculated values within each sector, expressed is a percentage, For example, in Fig. 9a, the numbers at the top center indicate an arlthmetic average exposure of $1.1 \times 10^{-10} \mathrm{~s} / \mathrm{m}^{3}$, with a range of exposures between $0.8 \times 10^{-10}$ and $1.4 \times 10^{-10}$. On all. the charts, tho bighest value in each sector is less than a factor of twe greater than the average $(+100 \%$ and $-50 \%$ are [actors of: two (rom the averige), Thase sectors with the larger percentages are genesally assoctated with the smaller exposure values during the winter season. Note that the highest annual exposures aro toward the NW and ESE for the arlthmet ic avernge and $5 \%$ values, respectively. During the winter, arithmet ic averages are greatest toward the NW and $\mathrm{N}$; $5 \%$ values show a maximun toward the $S$. In summer, maximum values occur toward the $\mathrm{SW}$ (artthmetis average) and the ESE and SW ( $5 \%$ values). Surface deposition values, in relative units of $\mathrm{m}^{-2}$, may be obtalned by dividing the exposures by $v_{d}(0.01 \mathrm{~m} / \mathrm{s})$.

Table 15 gives the $r$ lative exposures $\left(s / t^{3}\right)$ at the site boundary along the critical azimuth and at the closest boundary from hrea 410 , in the event of a 100-ton fission explosion. Table 16 presents relative exposures at various distances along the critical anfmuth, Note the unformity of the althmetic averages at froth 1 to $100 \mathrm{~km}$. 
Table 15. Relative exposures ${ }^{a}$ at stte boundary along the critical azimuth and at the closest boundary for a 100-ton Elssion explosion.

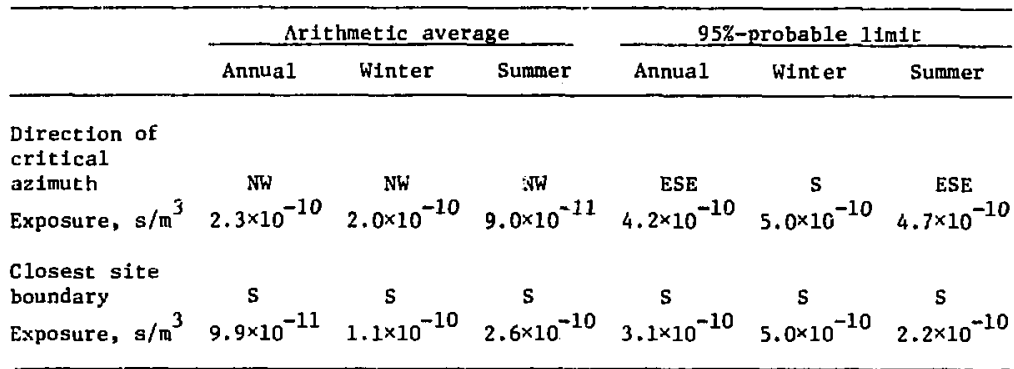

${ }^{a}$ Deposition values $\left(\ln \mathrm{m}^{-2}\right)$ are $1 / 100$ of exposure values.

Table 16. Relative exposidres (in unitg of $10^{-10} \mathrm{~s} / \mathrm{m}^{3}$ ) at various distances along the critical aa Imuth for a 100-ton fission explosion.

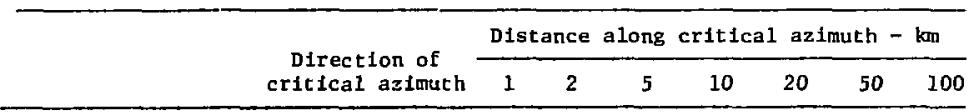

Annual exposure

$\begin{array}{lllllllll}\text { arithmetic average } & \text { NW } & 2.0 & 2.0 & 1.9 & 1.8 & 1.9 & 2.5 & 3.4 \\ 95 \%-p r o b a b l e ~ l i m i t & \text { ESE } & 4.3 & 4.2 & 4.1 & 4.0 & 4.2 & 5.7 & 8.2\end{array}$

Winter exposure

$\begin{array}{lllllllll}\text { arithmetic average } & \mathrm{NW} & 2.2 & 2.1 & 1.9 & 1.7 & 1.6 & 1.7 & 2.1 \\ 95 \% \text {-probable 11mit } & \mathrm{S} & 5.3 & 5.2 & 5.1 & 5.0 & 5.2 & 6.4 & 8.7\end{array}$

Summer exposure

$\begin{array}{lllllllll}\text { ar1thuetic average } & \text { NW } & 2.0 & 1.9 & 1.8 & 1.8 & 2.0 & 2.8 & 3.7 \\ 95 \%-p r o b a b 1 e ~ 11 m i t & \text { ESE } & 4.3 & 4.2 & 4.2 & 4.2 & 4.7 & 6.6 & 1.0\end{array}$




\section{References}

1. R. F. Quiring, Climatologica 1 Data, Nevada Test Site and Nuclear Rocket Developnenc Station, ESSA Research Laboratories, Las Vegas, Nev., Technical Memorandum ERLTM-ARL7, (1968).

2. J. R. McDonald, J. E. Minor, and K. C. Mehta, Development of a Design Basis Tornado and Structural Design Criterla for the Hevada Test Site, Nevada, Lawrence Livermore Laboratory Rept. UCRL-13668 (1975).

3. R. F. Quiring, Wind Summaries for Area 410 Basin NW Stat private communtcation (April 26, 1972).

4. D. H. Slade, Ed., Meteorology and Atomic Energy 1968, U.S. Atomic Energy Commission, New Brunswick, New Jersey, Rept. T10-24190 (1968).

5. W. C. Hardwick, Monthly Weather Rev. 101 (10), 763 (1963).

6. K. R. Peterson, "IPS: A Computer Program for Calculating Diffusion and Deposition for an Instantaneous Point Source," Lawrence Livermore Laboratory private communication (1976).

7. J. J. Walton, J. App1. Meteor. 12 (3), 547 (1973). 